\title{
Influence of Hall Current and Thermal Radiation on MHD Convective Heat and Mass Transfer in a Rotating Porous Channel with Chemical Reaction
}

\author{
Dulal Pal ${ }^{1}$ and Babulal Talukdar ${ }^{2}$ \\ ${ }^{1}$ Department of Mathematics, Visva-Bharati University, Santiniketan, West Bengal 731235, India \\ ${ }^{2}$ Department of Mathematics, Gobindapur High School, Kalabagh, Murshidabad, West Bengal 742213, India \\ Correspondence should be addressed to Dulal Pal; dulalp123@rediffmail.com
}

Received 14 February 2013; Accepted 17 June 2013

Academic Editor: Song Cen

Copyright (C) 2013 D. Pal and B. Talukdar. This is an open access article distributed under the Creative Commons Attribution License, which permits unrestricted use, distribution, and reproduction in any medium, provided the original work is properly cited.

\begin{abstract}
A theoretical study is carried out to obtain an analytic solution of heat and mass transfer in a vertical porous channel with rotation and Hall current. A constant suction and injection is applied to the two insulating porous plates. A strong magnetic field is applied in the transverse direction. The entire system rotates with uniform angular velocity $\Omega$ about the axis normal to the plates. The governing equations are solved by perturbation technique to obtain the analytical results for velocity, temperature, and concentration fields and shear stresses. The steady and unsteady resultant velocities along with the phase differences for various values of physical parameters are discussed in detail. The effects of rotation, buoyancy force, magnetic field, thermal radiation, and heat generation parameters on resultant velocity, temperature, and concentration fields are analyzed.
\end{abstract}

\section{Introduction}

Free convection in channel flow has many important applications in designing ventilating and heating of buildings, cooling of electronic components of a nuclear reactor, bed thermal storage, and heat sink in the turbine blades. Convective flows driven by temperature difference of the bounding walls of channels are important in industrial applications. ElHakiem [1] studied the unsteady MHD oscillatory flow on free convection radiation through a porous medium with a vertical infinite surface that absorbs the fluid with a constant velocity. Jaiswal and Soundalgekar [2] analyzed the effects of suction with oscillating temperature on a flow past an infinite porous plate. Singh et al. [3] studied the unsteady free convective flow in a porous medium bounded by an infinite vertical porous plate in the presence of rotation. Pal and Shivakumara [4] studied the mixed convection heat transfer from a vertical plate in a porous medium.

Hydromagnetic convection with heat transfer in a rotating medium has important applications in MHD generators and accelerators design, geophysics, and nuclear power reactors. MHD free convection and mass transfer flows in a rotating system have diverse applications. The effects of Hall currents cannot be neglected as the conducting fluid when it is an ionized gas, and applied field strength is strong then the electron cyclotron frequency $\omega=e B / m$ (where $e$, $B$, and $m$ denote the electron charge, the applied magnetic field, and mass of an electron, resp.) exceeds the collision frequency so that the electron makes cyclotron orbit between the collisions which will divert in a direction perpendicular to the magnetic and electric fields directions. Thus, if an electric field is applied perpendicular to the magnetic field then whole current will not pass along the electric field. This phenomena of flow of the electric current across an electric field with magnetic field is known as Hall effect, and accordingly this current is known as Hall current [5]. Thus, it is essential to analyze the effects of Hall currents in many industrial problems. Gupta [6] has studied the influence of Hall current on steady MHD flow in a viscous fluid. Jana et al. [7] analyzed the hall effect in steady flow past an infinite porous flat plate. Makinde and Mhone [8] studied hydromagnetic oscillatory flow through a channel having porous medium. Zhang and Wang [9] analyzed the effect of magnetic field in a power-law fluid over a vertical stretching sheet. Hameed 
and Nadeem [10] analyzed unsteady hydromagnetic flow of a non-Newtonian fluid over a porous plate. Makinde et al. [11] examined the effect of magnetic field in a rotating porous medium cylindrical annulus. Sibanda and Makinde [12] analyzed effects of magnetic fields on heat transfer on a rotating disk in a porous medium with Ohmic heating and viscous dissipation. Pop and Watanabe [13] analyzed convective flow of a conducting fluid in the presence of magnetic field and Hall current. Saha et al. [14] studied Hall current effect on MHD natural convection flow from vertical flat plate. Recently, Pal et al. [15] examined the influence of Hall current and chemical reaction on oscillatory mixed convection radiation of a micropolar fluid in a rotating system.

Radiation effects on free convection flow have become very important due to its applications in space technology, processes having high temperature, and design of pertinent equipments. Moreover, heat and mass transfer with thermal radiation on convective flows is very important due its significant role in the surface heat transfer. Recent developments in gas cooled nuclear reactors, nuclear power plants, gas turbines, space vehicles, and hypersonic flights have attracted research in this field. The unsteady convective flow in a moving plate with thermal radiation were examined by Cogley et al. [16] and Mansour [17]. The combined effects of radiation and buoyancy force past a vertical plate were analyzed by Hossain and Takhar [18]. Hossain et al. [19] analyzed the influence of thermal radiation on convective flows over a porous vertical plate. Seddeek [20] explained the importance of thermal radiation and variable viscosity on unsteady forced convection with an align magnetic field. Muthucumaraswamy and Senthil [21] studied the effects of thermal radiation on heat and mass transfer over a moving vertical plate. Pal [22] investigated convective heat and mass transfer in a stagnation-point flow towards a stretching sheet with thermal radiation. Aydin and Kaya [23] justified the effects of thermal radiation on mixed convection flow over a permeable vertical plate with magnetic field. Mohamed [24] studied unsteady MHD flow over a vertical moving porous plate with heat generation and Soret effect. Chauhan and Rastogi [25] analyzed the effects of thermal radiation, porosity, and suction on unsteady convective hydromagnetic vertical rotating channel. Ibrahim and Makinde [26] investigated radiation effect on chemically reaction MHD boundary layer flow of heat and mass transfer past a porous vertical flat plate. Pal and Mondal [27] studied the effects of thermal radiation on MHD Darcy-Forchheimer convective flow past a stretching sheet in a porous medium. Palani and Kim [28] analyzed the effect of thermal radiation on convection flow past a vertical cone with surface heat flux. Recently, Mahmoud and Waheed [29] examined thermal radiation on flow over an infinite flat plate with slip velocity.

The study of heat and mass transfer due to chemical reaction is also very importance because of its occurrence in most of the branches of science and technology. The processes involving mass transfer effects are important in chemical processing equipments which are designed to draw high value products from cheaper raw materials with the involvement of chemical reaction. In many industrial processes, the species undergo some kind of chemical reaction with the ambient fluid which may affect the flow behaviour and the production quality of final products. Aboeldahab and Elbarbary [30] examined heat and mass transfer over a vertical plate in the presence of magnetic field and Hall effect. Abo-Eldahab and El Aziz [31] investigated the Hall current and Joule heating effects on electrically conducting fluid past a semi-infinite plate with strong magnetic field and heat generation/absorption. Kandasamy et al. [32] discussed the effects of chemical reaction and magnetic field on heat and mass transfer over a vertical stretching surface. Muthucumaraswamy and Janakiraman [33] analyzed the effects of mass transfer over a vertical oscillating plate with chemical reaction. Sharma and Singh [34] have analyzed the unsteady MHD free convection flow and heat transfer over a vertical porous plate in the presence of internal heat generation and variable suction. Sudheer Babu and Satya Narayan [35] examined chemical reaction and thermal radiation effects on MHD convective flow in a porous medium in the presence of suction. Makinde and Chinyoka [36] studied the effects of magnetic field on MHD Couette flow of a third-grade fluid with chemical reaction. Recently, Pal and Talukdar [37] investigated the influence of chemical reaction and Joule heating on unsteady convective viscous dissipating fluid over a vertical plate in porous media with thermal radiation and magnetic field.

The objective of the present study is to analyze the effects of Hall current, thermal radiation, and first-order chemical reaction on the oscillatory convective flow and mass transfer with suction injection in a rotating vertical porous channel. The present results are compared with those of Singh and Kumar [38], and a very good agreement is found.

\section{Problem Formulation}

We consider unidirectional oscillatory free convective flow of a viscous incompressible and electrically conducting fluid between two insulating infinite vertical permeable plates separated by a distance $d$. A constant injection velocity $w_{0}$ is applied at the stationary plate $z^{*}=0$. Also, a constant suction velocity $w_{0}$ is applied at the plate $z^{*}=d$, which oscillates in its own plane with a velocity $U^{*}\left(t^{*}\right)$ about a nonzero constant mean velocity $U_{0}$. The channel rotates as a rigid body with angular velocity $\Omega^{*}$ about the $z^{*}$-axis perpendicular to the planes of the plates. A strong transverse magnetic field of uniform strength $H_{0}$ is applied along the axis of rotation by neglecting induced electric and magnetic fields. The fluid is assumed to be a gray, emitting, and absorbing, but nonscattering medium. The radiative heat flux term can be simplified by using the Rosseland approximation. It is also assumed that the chemically reactive species undergo firstorder irreversible chemical reaction.

The solenoidal relation for the magnetic field $\nabla \cdot \vec{H}=$ 0 , where $\vec{H}=\left(H_{x}^{*}, H_{y}^{*}, H_{z}^{*}\right)$ gives $H_{z}^{*}=H_{0}$ (constant) everywhere in the flow field, which gives $\vec{H}=\left(0,0, H_{0}\right)$. If $\left(J_{x}^{*}, J_{y}^{*}, J_{z}^{*}\right)$ are the component of electric current density $\vec{J}$, then the equation of conservation of electric charge $\nabla \cdot \vec{J}=0$ gives $J_{z}^{*}=$ constant. This constant is zero, that is, $J_{z}^{*}=0$ 
everywhere in the flow since the plate is electrically nonconducting. The generalized Ohm's law, in the absence of the electric field [39], is of the form

$$
\vec{J}+\frac{\omega_{e} \tau_{e}}{H_{0}}(\vec{J} \times \vec{H})=\sigma\left(\mu_{e} \vec{V} \times \vec{H}+\frac{1}{e n_{e}} \nabla p_{e}\right),
$$

where $\vec{V}, \sigma, \mu_{e}, \omega_{e}, \tau_{e}, e, n_{e}$, and $p_{e}$ are the velocity, the electrical conductivity, the magnetic permeability, the cyclotron frequency, the electron collision time, the electric charge, the number density of the electron, and the electron pressure, respectively. Under the usual assumption, the electron pressure (for a weakly ionized gas), the thermoelectric pressure, and ion slip are negligible, so we have from the Ohm's law

$$
\begin{gathered}
J_{x}^{*}+\omega_{e} \tau_{e} J_{y}^{*}=\sigma \mu_{e} H_{0} v^{*} \\
J_{y}^{*}-\omega_{e} \tau_{e} J_{x}^{*}=-\sigma \mu_{e} H_{0} u^{*},
\end{gathered}
$$

from which we obtain that

$$
J_{x}^{*}=\frac{\sigma \mu_{e} H_{0}\left(m u^{*}+v^{*}\right)}{1+m^{2}}, \quad J_{y}^{*}=\frac{\sigma \mu_{e} H_{0}\left(m v^{*}-u^{*}\right)}{1+m^{2}} .
$$

Since the plates are infinite in extent, all the physical quantities except the pressure depend only on $z^{*}$ and $t^{*}$. The physical configuration of the problem is shown in Figure 1. A Cartesian coordinate system is assumed, and $z^{*}$-axis is taken normal to the plates, while $x^{*}$ - and $y^{*}$-axes are in the upward and perpendicular directions on the plate $z^{*}=0$ (origin), respectively. The velocity components $u^{*}, v^{*}, w^{*}$ are in the $x^{*}-, y^{*}-, z^{*}$-directions, respectively. The governing equations in the rotating system in presence of Hall current, thermal radiation, and chemical reaction are given by the following equations:

$$
\begin{gathered}
\frac{\partial w^{*}}{\partial z^{*}}=0 \Longrightarrow w^{*}=w_{0}, \\
\frac{\partial u^{*}}{\partial t^{*}}+w_{0} \frac{\partial u^{*}}{\partial z^{*}}-2 \Omega^{*} v^{*}=-\frac{1}{\rho} \frac{\partial P^{*}}{\partial x^{*}}+\nu \frac{\partial^{2} u^{*}}{\partial z^{* 2}}+g_{0} \beta\left(T^{*}-T_{d}\right) \\
+g_{0} \beta^{*}\left(C^{*}-C_{d}\right)+\frac{H_{0}}{\rho} J_{y}^{*}
\end{gathered}
$$$$
\frac{\partial v^{*}}{\partial t^{*}}+w_{0} \frac{\partial v^{*}}{\partial z^{*}}+2 \Omega^{*} u^{*}=-\frac{1}{\rho} \frac{\partial P^{*}}{\partial y^{*}}+\nu \frac{\partial^{2} v^{*}}{\partial z^{* 2}}-\frac{H_{0}}{\rho} J_{x}^{*},
$$$$
\frac{\partial T^{*}}{\partial t^{*}}+w_{0} \frac{\partial T^{*}}{\partial z^{*}}=\frac{\kappa}{\rho c_{p}} \frac{\partial^{2} T^{*}}{\partial z^{* 2}}-\frac{Q_{0}}{\rho c_{p}}\left(T^{*}-T_{d}\right)-\frac{1}{\rho c_{p}} \frac{\partial q_{r}^{*}}{\partial z^{*}},
$$$$
\frac{\partial C^{*}}{\partial t^{*}}+w_{0} \frac{\partial C^{*}}{\partial z^{*}}=D_{m} \frac{\partial^{2} C^{*}}{\partial z^{* 2}}-k_{1}\left(C^{*}-C_{d}\right)
$$

where $m\left(=\omega_{e} \tau_{e}\right)$ is the Hall parameter, $\beta$ and $\beta^{*}$ are the coefficients of thermal and solutal expansion, $c_{p}$ is the specific heat at constant pressure, $\rho$ is the density of the fluid, $v$ is the

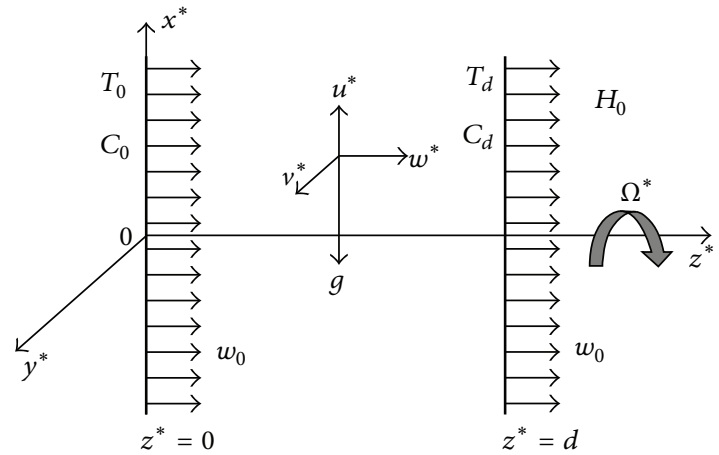

Figure 1: Physical configuration of the problem.

kinematics viscosity, $\kappa$ is the fluid thermal conductivity, $g_{0}$ is the acceleration of gravity, $Q_{0}$ is the additional heat source, $q_{r}^{*}$ is the radiative heat flux, $D_{m}$ is the molecular diffusivity, $k_{1}$ is the chemical reaction rate constant. The radiative heat flux is given by $q_{r}^{*}=-\left(4 \sigma^{*} / 3 k^{*}\right)\left(\partial T^{* 4} / \partial z^{*}\right)$, in which $\sigma^{*}$ and $k^{*}$ are the Stefan-Boltzmann constant and the mean absorption coefficient, respectively.

The initial and boundary conditions as suggested by the physics of the problem are

$$
\begin{aligned}
& u^{*}=v^{*}=0, \quad T^{*}=T_{0}+\epsilon\left(T_{0}-T_{d}\right) \cos \omega^{*} t^{*}, \\
& C^{*}=C_{0}+\epsilon\left(C_{0}-C_{d}\right) \cos \omega^{*} t^{*} \quad \text { at } z^{*}=0 \\
& u^{*}=U^{*}\left(t^{*}\right)=U_{0}\left(1+\epsilon \cos \omega^{*} t^{*}\right), \quad v^{*}=0, \\
& T^{*}=T_{d}, \quad C^{*}=C_{d} \quad \text { at } z^{*}=d,
\end{aligned}
$$

where $\epsilon$ is a small constant.

We now introduce the dimensionless variables as follows:

$$
\begin{gathered}
\eta=\frac{z^{*}}{d}, \quad u=\frac{u^{*}}{U_{0}}, \quad v=\frac{v^{*}}{U_{0}}, \quad t=\frac{\omega^{*}}{t^{*}}, \\
\omega=\frac{\omega^{*} d^{2}}{v}, \quad \Omega=\frac{\Omega^{*} d^{2}}{v}, \quad \lambda=\frac{w_{0} d}{v}, \\
\theta=\frac{T^{*}-T_{d}}{T_{0}-T_{d}}, \quad \phi=\frac{C^{*}-C_{d}}{C_{0}-C_{d}} .
\end{gathered}
$$

After combining (5) and (6) and taking $q=u+i v$, then (5)(8) reduce to

$$
\begin{aligned}
& \omega \frac{\partial q}{\partial t}+\lambda \frac{\partial q}{\partial \eta}= \frac{\partial^{2} q}{\partial \eta^{2}}+w \frac{\partial U}{\partial t}-2 i \Omega(q-U) \\
&-\frac{M^{2}(1+i m)}{1+m^{2}}(q-U)+\lambda^{2}(\mathrm{Gr} \theta+\mathrm{Gm} \phi) \\
& \omega \frac{\partial \theta}{\partial t}+\lambda \frac{\partial \theta}{\partial \eta}=\frac{1}{\operatorname{Pr}}\left(1+\frac{4}{3 R}\right) \frac{\partial^{2} q}{\partial \eta^{2}}-\frac{Q_{H}}{\operatorname{Pr}} \theta \\
& \omega \frac{\partial \phi}{\partial t}+\lambda \frac{\partial \phi}{\partial \eta}=\frac{1}{\mathrm{Sc}} \frac{\partial^{2} \phi}{\partial \eta^{2}}-\xi \phi
\end{aligned}
$$


where $\mathrm{Gr}=g_{0} \beta \nu\left(T 0_{w}-T_{d}\right) / U_{0} w_{0}^{2}$ is the modified thermal Grashof number, $\mathrm{Gm}=g_{0} \beta^{*} \nu\left(C_{0}-C_{d}\right) / U_{0} w_{0}^{2}$ is the modified solutal Grashof number, $\operatorname{Pr}=\nu \rho c_{p} / \kappa$ is the Prandtl number, $M=H_{0} d \sqrt{\sigma / \mu}$ is the Hartmann number, $Q_{H}=Q_{0} d^{2} / \kappa$ is the heat source parameter, $R=\kappa k^{*} / 4 \sigma^{*} T_{d}$ is the radiation parameter, $\mathrm{Sc}=\nu / D m$ is the Schmidt number, and $\xi=$ $k_{1} d^{2} / \nu$ is the reaction parameter. form as

The boundary conditions (9) can be expressed in complex

$$
\begin{gathered}
q=0, \quad \theta=1+\frac{\epsilon}{2}\left(e^{i t}+e^{-i t}\right), \quad \phi=1+\frac{\epsilon}{2}\left(e^{i t}+e^{-i t}\right) \\
\text { at } \eta=0, \\
q=U(t)=1+\frac{\epsilon}{2}\left(e^{i t}+e^{-i t}\right), \quad \theta=0, \quad \phi=0 \quad \text { at } \eta=1 .
\end{gathered}
$$

\section{Method of Solution}

The set of partial differential equations (11) cannot be solved in closed form. So it is solved analytically after these equations are reduced to a set of ordinary differential equations in dimensionless form. We assume that

$$
\mathfrak{R}(\eta, t)=\mathfrak{R}_{0}(\eta)+\frac{\epsilon}{2}\left(\mathfrak{R}_{1}(\eta) e^{i t}+\mathfrak{R}_{2}(\eta) e^{-i t}\right),
$$

where $\mathfrak{R}$ stands for $q$ or $\theta$ or $\phi$, and $\epsilon \ll 1$ which is a perturbation parameter. The method of solution is applicable for small perturbation.

Substituting (13) into (11) and comparing the harmonic and nonharmonic terms, we obtain the following ordinary differential equations:

$$
\begin{aligned}
& q_{0}^{\prime \prime}-\lambda q_{0}^{\prime}-S q_{0}=-S-\lambda^{2}\left(\mathrm{Gr} \theta_{0}+\mathrm{Gm} \phi_{0}\right), \\
& q_{1}^{\prime \prime}-\lambda q_{1}^{\prime}-(S+i \omega) q_{1}=-(S+i \omega) \\
& -\lambda^{2}\left(\mathrm{Gr} \theta_{1}+\mathrm{Gm} \phi_{1}\right) \\
& q_{2}^{\prime \prime}-\lambda q_{2}^{\prime}-(S-i \omega) q_{1}=-(S-i \omega) \\
& -\lambda^{2}\left(\mathrm{Gr} \theta_{2}+\mathrm{Gm} \phi_{2}\right) \text {, } \\
& \theta_{0}^{\prime \prime}-\frac{3 \lambda \operatorname{Pr} R}{3 R+4} \theta_{0}^{\prime}-\frac{3 R Q_{H}}{3 R+4} \theta_{0}=0, \\
& \theta_{1}^{\prime \prime}-\frac{3 \lambda \operatorname{Pr} R}{3 R+4} \theta_{1}^{\prime}-\frac{3 R}{3 R+4}\left(i \omega \operatorname{Pr}+Q_{H}\right) \theta_{1}=0, \\
& \theta_{2}^{\prime \prime}-\frac{3 \lambda \operatorname{Pr} R}{3 R+4} \theta_{2}^{\prime}+\frac{3 R}{3 R+4}\left(i \omega \operatorname{Pr}-Q_{H}\right) \theta_{2}=0, \\
& \phi_{0}^{\prime \prime}-\operatorname{Sc} \lambda \phi_{0}^{\prime}-\operatorname{Sc} \xi \phi_{0}=0, \\
& \phi_{1}^{\prime \prime}-\operatorname{Sc} \lambda \phi_{1}^{\prime}-\operatorname{Sc}(i \omega+\xi) \phi_{1}=0, \\
& \phi_{2}^{\prime \prime}-\operatorname{Sc} \lambda \phi_{2}^{\prime}+\operatorname{Sc}(i \omega-\xi) \phi_{2}=0,
\end{aligned}
$$

where $S=\left(M^{2}(1+i m) /\left(1+m^{2}\right)\right)+2 i \Omega$ and dashes denote the derivatives w.r.t. $\eta$.
The transformed boundary conditions are

$$
\begin{array}{ll}
q_{0}=0, & q_{1}=0, \quad q_{2}=0, \\
\theta_{0}=1, & \theta_{1}=1, \quad \theta_{2}=1, \\
\phi_{0}=1, & \phi_{1}=1, \quad \phi_{2}=1 \quad \text { at } \eta=0, \\
q_{0}=1, & q_{1}=1, \quad q_{2}=1, \\
\theta_{0}=0, & \theta_{1}=0, \quad \theta_{2}=0, \\
\phi_{0}=0, & \phi_{1}=0, \quad \phi_{2}=0 \quad \text { at } \eta=1 .
\end{array}
$$

The solutions of (14) under the boundary conditions (15) are

$$
\begin{aligned}
& q_{0}=1-e^{h_{14} \eta}+A_{1}\left(e^{h_{14} \eta}-e^{h_{8} \eta}\right)-A_{2}\left(e^{h_{14} \eta}-e^{h_{7} \eta}\right) \\
& +A_{3}\left(e^{h_{14} \eta}-e^{h_{2} \eta}\right)-A_{4}\left(e^{h_{14} \eta}-e^{h_{1} \eta}\right)+\frac{e^{h_{13} \eta}-e^{h_{14} \eta}}{e^{h_{13}}-e^{h_{14}}} \\
& \times\left[e^{h_{14}}-A_{1}\left(e^{h_{14}}-e^{h_{8}}\right)+A_{2}\left(e^{h_{14}}-e^{h_{7}}\right)\right. \\
& \left.-A_{3}\left(e^{h_{14}}-e^{h_{2}}\right)+A_{4}\left(e^{h_{14}}-e^{h_{1}}\right)\right], \\
& q_{1}=1-e^{h_{16} \eta}+A_{5}\left(e^{h_{16} \eta}-e^{h_{10} \eta}\right)-A_{6}\left(e^{h_{16} \eta}-e^{h_{9} \eta}\right) \\
& +A_{7}\left(e^{h_{16} \eta}-e^{h_{4} \eta}\right)-A_{8}\left(e^{h_{16} \eta}-e^{h_{3} \eta}\right)+\frac{e^{h_{15} \eta}-e^{h_{16} \eta}}{e^{h_{15}}-e^{h_{16}}} \\
& \times\left[e^{h_{16}}-A_{5}\left(e^{h_{16}}-e^{h_{10}}\right)+A_{6}\left(e^{h_{16}}-e^{h_{9}}\right)\right. \\
& \left.-A_{7}\left(e^{h_{16}}-e^{h_{4}}\right)+A_{8}\left(e^{h_{16}}-e^{h_{3}}\right)\right], \\
& q_{2}=1-e^{h_{18} \eta}+A_{9}\left(e^{h_{18} \eta}-e^{h_{12} \eta}\right)-A_{10}\left(e^{h_{18} \eta}-e^{h_{11} \eta}\right) \\
& +A_{11}\left(e^{h_{18} \eta}-e^{h_{6} \eta}\right)-A_{12}\left(e^{h_{18} \eta}-e^{h_{5} \eta}\right)+\frac{e^{h_{17} \eta}-e^{h_{18} \eta}}{e^{h_{17}}-e^{h_{18}}} \\
& \times\left[e^{h_{18}}-A_{9}\left(e^{h_{18}}-e^{h_{12}}\right)+A_{10}\left(e^{h_{18}}-e^{h_{11}}\right)\right. \\
& \left.-A_{11}\left(e^{h_{18}}-e^{h_{6}}\right)+A_{12}\left(e^{h_{18}}-e^{h_{5}}\right)\right], \\
& \theta_{0}=\frac{e^{h_{7}+h_{8} \eta}-e^{h_{8}+h_{7} \eta}}{e^{h_{7}}-e^{h_{8}}}, \\
& \theta_{1}=\frac{e^{h_{9}+h_{10} \eta}-e^{h_{10}+h_{9} \eta}}{e^{h_{9}}-e^{h_{10}}}, \\
& \theta_{2}=\frac{e^{h_{11}+h_{12} \eta}-e^{h_{12}+h_{11} \eta}}{e^{h_{11}}-e^{h_{12}}}, \\
& \phi_{0}=\frac{e^{h_{1}+h_{2} \eta}-e^{h_{2}+h_{1} \eta}}{e^{h_{1}}-e^{h_{2}}},
\end{aligned}
$$




$$
\begin{aligned}
& \phi_{1}=\frac{e^{h_{3}+h_{4} \eta}-e^{h_{4}+h_{3} \eta}}{e^{h_{3}}-e^{h_{4}}}, \\
& \phi_{2}=\frac{e^{h_{5}+h_{6} \eta}-e^{h_{6}+h_{5} \eta}}{e^{h_{5}}-e^{h_{6}}} .
\end{aligned}
$$

\section{Amplitude and Phase Difference due to Steady and Unsteady Flow}

Equation (16) corresponds to the steady part, which gives $u_{0}$ as the primary and $v_{0}$ as secondary velocity components. The amplitude (resultant velocity) and phase difference due to these primary and secondary velocities for the steady flow are given by

$$
R_{0}=\sqrt{u_{0}^{2}+v_{0}^{2}}, \quad \alpha_{0}=\tan ^{-1}\left(\frac{v_{0}}{u_{0}}\right)
$$

where $u_{0}(\eta)+i v_{0}(\eta)=q_{0}(\eta)$.

Equations (17) and (18) together give the unsteady part of the flow. Thus, unsteady primary and secondary velocity components $u_{1}(\eta)$ and $v_{1}(\eta)$, respectively, for the fluctuating flow can be obtained from the following:

$$
\begin{aligned}
u_{1}(\eta, t)= & {\left[\operatorname{Real} q_{1}(\eta)+\operatorname{Real} q_{2}(\eta)\right] \cos t } \\
& -\left[\operatorname{Im} q_{1}(\eta)-\operatorname{Im} q_{2}(\eta)\right] \sin t \\
v_{1}(\eta, t)= & {\left[\operatorname{Real} q_{1}(\eta)-\operatorname{Real} q_{2}(\eta)\right] \sin t } \\
& +\left[\operatorname{Im} q_{1}(\eta)+\operatorname{Im} q_{2}(\eta)\right] \cos t
\end{aligned}
$$

The amplitude (resultant velocity) and the phase difference of the unsteady flow are given by

$$
R_{v}=\sqrt{u_{1}^{2}+v_{1}^{2}}, \quad \alpha_{1}=\tan ^{-1}\left(\frac{v_{1}}{u_{1}}\right)
$$

where $u_{1}(\eta)+i v_{1}(\eta)=q_{1}(\eta) e^{i t}+q_{2}(\eta) e^{-i t}$.

The amplitude (resultant velocity) and the phase difference

$$
R_{n}=\sqrt{u^{2}+v^{2}}, \quad \alpha=\tan ^{-1}\left(\frac{v}{u}\right)
$$

where $u=$ Real part of $q$ and $v=$ Imaginary part of $q$.

\section{Amplitude and Phase Difference of Shear Stresses due to Steady and Unsteady Flow at the Plate}

The amplitude and phase difference of shear stresses at the stationary plate $(\eta=0)$ for the steady flow can be obtained as

$$
\tau_{0 r}=\sqrt{\tau_{0 x}^{2}+\tau_{0 y}^{2}}, \quad \beta_{0}=\tan ^{-1}\left(\frac{\tau_{0 y}}{\tau_{0 x}}\right)
$$

where

$$
\begin{aligned}
\left(\frac{\partial q_{0}}{\partial \eta}\right)_{\eta=0}= & \tau_{0 x}+i \tau_{0 y}=-h_{14}+A_{1}\left(h_{14}-h_{8}\right)-A_{2}\left(h_{14}-h_{7}\right) \\
& +A_{3}\left(h_{14}-h_{2}\right)-A_{4}\left(h_{14}-h_{1}\right)+\frac{h_{13}-h_{14}}{e^{h_{13}}-e^{h_{14}}} \\
& \times\left[e^{h_{14}}-A_{1}\left(e^{h_{14}}-e^{h_{8}}\right)+A_{2}\left(e^{h_{14}}-e^{h_{7}}\right)\right. \\
& \left.-A_{3}\left(e^{h_{14}}-e^{h_{2}}\right)+A_{4}\left(e^{h_{14}}-e^{h_{1}}\right)\right] .
\end{aligned}
$$

For the unsteady part of flow, the amplitude and phase difference of shear stresses at the stationary plate $(\eta=0)$ can be obtained as

$$
\tau_{1 r}=\sqrt{\tau_{1 x}^{2}+\tau_{1 y}^{2}}, \quad \beta_{1}=\tan ^{-1}\left(\frac{\tau_{1 y}}{\tau_{1 x}}\right),
$$

where

$$
\begin{aligned}
\tau_{1 x}+ & i \tau_{1 y}=\left(\frac{\partial q_{1}}{\partial \eta}\right)_{\eta=0} e^{i t}+\left(\frac{\partial q_{2}}{\partial \eta}\right)_{\eta=0} e^{-i t} \\
\left(\frac{\partial q_{1}}{\partial \eta}\right)_{\eta=0}= & -h_{16}+A_{5}\left(h_{16}-h_{10}\right)-A_{6}\left(h_{16}-h_{9}\right) \\
& +A_{7}\left(h_{16}-h_{4}\right)-A_{8}\left(h_{16}-h_{3}\right)+\frac{h_{15}-h_{16}}{e^{h_{15}}-e^{h_{16}}} \\
& \times\left[e^{h_{16}}-A_{5}\left(e^{h_{16}}-e^{h_{10}}\right)+A_{6}\left(e^{h_{16}}-e^{h_{9}}\right)\right. \\
& \left.-A_{7}\left(e^{h_{16}}-e^{h_{4}}\right)+A_{8}\left(e^{h_{16}}-e^{h_{3}}\right)\right] \\
\left(\frac{\partial q_{2}}{\partial \eta}\right)_{\eta=0} & -h_{18}+A_{9}\left(h_{18}-h_{12}\right)-A_{10}\left(h_{18}-h_{11}\right) \\
& +A_{11}\left(h_{16}-h_{6}\right)-A_{12}\left(h_{18}-h_{5}\right)+\frac{h_{17}-h_{18}}{e^{h_{17}}-e^{h_{18}}} \\
& \times\left[e^{h_{18}}-A_{9}\left(e^{h_{18}}-e^{h_{12}}\right)+A_{10}\left(e^{h_{18}}-e^{h_{11}}\right)\right. \\
& \left.\quad-A_{11}\left(e^{h_{18}}-e^{h_{6}}\right)+A_{12}\left(e^{h_{18}}-e^{h_{5}}\right)\right]
\end{aligned}
$$

The amplitude and phase difference of shear stresses at the stationary plate $(\eta=0)$ for the flow can be obtained as

$$
\tau=\left(\frac{\partial q}{\partial \eta}\right)_{\eta=0}=\sqrt{\tau_{x}^{2}+\tau_{y}^{2}}, \quad \beta_{2}=\tan ^{-1}\left(\frac{\tau_{y}}{\tau_{x}}\right),
$$

where $\tau_{x}=$ Real part of $(\partial q / \partial \eta)_{\eta=0}$ and $\tau_{y}=$ Imaginary part of $(\partial q / \partial \eta)_{\eta=0}$.

The Nusselt number

$$
\mathrm{Nu}=-\left(1+\frac{4}{3 R}\right)\left(\frac{\partial \theta}{\partial \eta}\right)_{\eta=0}=N_{x}+i N_{y} .
$$

The rate of heat transfer (i.e., heat flux) at the plate in terms of amplitude and phase is given by

$$
\Theta=\sqrt{N_{x}^{2}+N_{y}^{2}}, \quad \gamma=\tan ^{-1}\left(\frac{N_{y}}{N_{x}}\right) .
$$


The Sherwood number

$$
\mathrm{Sh}=\left(\frac{\partial \phi}{\partial \eta}\right)_{\eta=0}=M_{x}+i M_{y}
$$

The rate of mass transfer (i.e., mass flux) at the plate in terms of amplitude and phase is given by

$$
\Phi=\sqrt{M_{x}^{2}+M_{y}^{2}}, \quad \delta=\tan ^{-1}\left(\frac{M_{y}}{M_{x}}\right) .
$$

\section{Results and Discussion}

The system of ordinary differential equations (14) with boundary conditions (15) is solved analytically by employing the perturbation technique. The solutions are obtained for the steady and unsteady velocity fields from (16)-(18), temperature fields from (19)-(21), and concentration fields are given by (22)-(24). The effects of various parameters on the thermal, mass, and hydrodynamic behaviors of buoyancyinduced flow in a rotating vertical channel are studied. The results are presented graphically and in tabular form. Temperature of the heated wall (left wall) at $z^{*}=0$ is a function of time as given in the boundary conditions, and the cooled wall at $z^{*}=d$ is maintained at a constant temperature. Further, it is assumed that the temperature difference is small enough so that the density changes of the fluid in the system will be small. When the injection/suction parameter $\lambda$ is positive, fluid is injected through the hot wall into the channel and sucked out through the cold wall. The numerical results of the amplitude of the shear stresses and the phase difference of the shear stresses at the stationary plate $\left(z^{*}=0\right)$ for the steady and unsteady flow are presented in Table 1 . The effect of various physical parameters on flow, heat, concentration fields, skin-friction, Nusselt number, and Sherwood number are presented graphically in Figures 2-16.

Table 1 shows a comparative study of the present results of amplitude and phase difference of shear stresses for the steady flow with those of Singh and Kumar [38]. It is seen from this table that the present results coincide very well with those of Singh and Kumar [38]. This confirms that the present analytical solutions are correct and accurate. Further, it is observed from this table that the effects of increasing the value of thermal Grashof number Gr, magnetic field $M$, and injection/suction parameter $\lambda$ are to increase amplitude and decrease the phase difference of shear stresses for the steady flow, whereas reverse effect is found by increasing the Hall parameter, $m$. The effects of increasing the angular velocity $\Omega$ are to increase both amplitude and phase difference of shear stresses, whereas reverse effects are seen by increasing the values of the Prandtl number. The computed results $\tau_{0 r}, \tau_{1 r}$, $\beta_{0}, \beta_{1}$ for the present problem are provided in Table 2 for various values of $\mathrm{Gm}, R, Q_{H}, \mathrm{Sc}, \xi$, and $m$. It is seen from this table that the values of $\tau_{0 r}$ and $\tau_{1 r}$ increase whereas the values of $\beta_{0}$ and $\beta_{1}$ decrease with the increase of solutal Grashof number $\mathrm{Gm}$, but the effects are reversed with an increase in the Hall parameter $m$, that is, the value of $\beta_{0}$ and $\beta_{1}$ are increased whereas there is decrease in the values of $\tau_{0 r}$ and $\tau_{1 r}$. Also, it is found that the values of $\tau_{0 r}, \beta_{0} \beta_{1}$ increase with
TABLE 1: Comparison results for the resultant velocity or amplitude and the phase difference of the unsteady flow with Singh and Kumar [38] for different values of $\mathrm{Gr}, M, m, \lambda, \Omega, \mathrm{Pr}$, and $\mathrm{Gm}=0.0, R=\infty$, $\xi=0.0$, Sc $\rightarrow 0.0$ (in present problem).

\begin{tabular}{lllllllccc}
\hline Gr $M$ & $m$ & $\lambda$ & $\Omega$ & $\operatorname{Pr}$ & \multicolumn{2}{c}{ Singh and Kumar [38] } & \multicolumn{2}{c}{ Present results } \\
& & & & & $\tau_{0 r}$ & $\beta_{0}$ & $\tau_{0 r}$ & $\beta_{0}$ \\
\hline 5 & 2 & 1 & 0.5 & 10 & 0.71 & 4.5847 & 0.7279 & 4.5847 & 0.7279 \\
10 & 2 & 1 & 0.5 & 10 & 0.71 & 4.6515 & 0.6815 & 4.6515 & 0.6815 \\
5 & 4 & 1 & 0.5 & 10 & 0.71 & 5.2797 & 0.6395 & 5.2797 & 0.6395 \\
5 & 2 & 3 & 0.5 & 10 & 0.71 & 4.4862 & 0.7627 & 4.4862 & 0.7627 \\
5 & 2 & 1 & 1.0 & 10 & 0.71 & 4.6162 & 0.6229 & 4.6162 & 0.6229 \\
5 & 2 & 1 & 0.5 & 20 & 0.71 & 6.3323 & 0.7614 & 6.3323 & 0.7614 \\
5 & 2 & 1 & 0.5 & 40 & 0.71 & 8.8924 & 0.7783 & 8.8924 & 0.7783 \\
5 & 2 & 1 & 0.5 & 80 & 0.71 & 12.558 & 0.7857 & 12.558 & 0.7857 \\
5 & 2 & 1 & 0.5 & 10 & 7.0 & 4.5726 & 0.7198 & 4.5726 & 0.7198 \\
\hline
\end{tabular}

TABLE 2: Values of $\tau_{0 r}, \beta_{0}, \tau_{1 r}$, and $\beta_{1}$ for the reference values of $\mathrm{Gr}=$ 5.0, $M=2.0, \lambda=0.5, \Omega=10.0, \operatorname{Pr}=0.71$, and $\omega=5.0$ at $t=\pi / 4$.

\begin{tabular}{lccccccccc}
\hline $\mathrm{Gm}$ & $R$ & $Q_{H}$ & $\mathrm{Sc}$ & $\xi$ & $m$ & $\tau_{0 r}$ & $\beta_{0}$ & $\tau_{1 r}$ & $\beta_{1}$ \\
\hline 5.0 & 1.0 & 5.0 & 0.15 & 0.1 & 1.0 & 4.6540 & 0.6877 & 6.4123 & 0.7980 \\
10.0 & 1.0 & 5.0 & 0.15 & 0.1 & 1.0 & 4.7299 & 0.6437 & 6.4542 & 0.7485 \\
5.0 & 5.0 & 5.0 & 0.15 & 0.1 & 1.0 & 4.6543 & 0.6900 & 6.4094 & 0.8011 \\
5.0 & 1.0 & 10.0 & 0.15 & 0.1 & 1.0 & 4.6545 & 0.6908 & 6.4219 & 0.8010 \\
5.0 & 1.0 & 5.0 & 0.60 & 0.1 & 1.0 & 4.6534 & 0.6869 & 6.3805 & 0.8006 \\
5.0 & 1.0 & 5.0 & 0.15 & 1.0 & 1.0 & 4.6541 & 0.6880 & 6.4132 & 0.7983 \\
5.0 & 1.0 & 5.0 & 0.15 & 1.0 & 3.0 & 4.5464 & 0.7197 & 6.3064 & 0.8366 \\
\hline
\end{tabular}

an increase in the radiation parameter $R$. It is noted that the values of both $\tau_{0 r}$ and $\beta_{0}$ increase due to an increase in the heat source parameter $Q_{H}$ and chemical reaction parameter $\xi$, whereas the effects are reversed with the increase in the Schmidt number, that is, the values of $\tau_{0 r}, \tau_{1 r} \beta_{1}$ decrease with an increase in the Schmidt number. Also, it is found that the value of $\beta_{1}$ decreases with an increase in the heat source parameter $Q_{H}$, chemical reaction parameter $\xi$ and Schmidt number Sc.

The profiles for resultant velocity $R_{n}$ for the flow are shown in Figures 2-6 for suction/injection parameter $\lambda$ and for small and large values of rotation parameter $\Omega, \epsilon$, and $\eta$, respectively. From Figure 2, it is observed that the increase in the suction parameter $\lambda$ leads to an increase of $R_{n}$ within the stationary plates. Similar trend of $R_{n}$ profiles is seen by increasing the rotation parameter $\Omega$, that is, resultant velocity profiles increase with increase in the rotation parameter $\Omega$ (small values) as shown in Figure 3. However, the opposite effect occurs near the right wall for large values of $\Omega$ as shown in Figure 4. This effect is due to the rotation effects being more dominant near the walls, so when $\Omega$ reaches high values, the secondary velocity component $v$ decreases with increase in $\Omega$ while approaching to the right plate. From Figure 5, it is observed that the increase in the $\epsilon$ leads to an increase of $R_{n}$ within the stationary plates. From Figure 6 , it is seen that the resultant velocity profiles increases with increase in $\eta$; also it is observed that the velocity oscillates with increasing time. The phase difference $\alpha$ for the flow is shown graphically in 


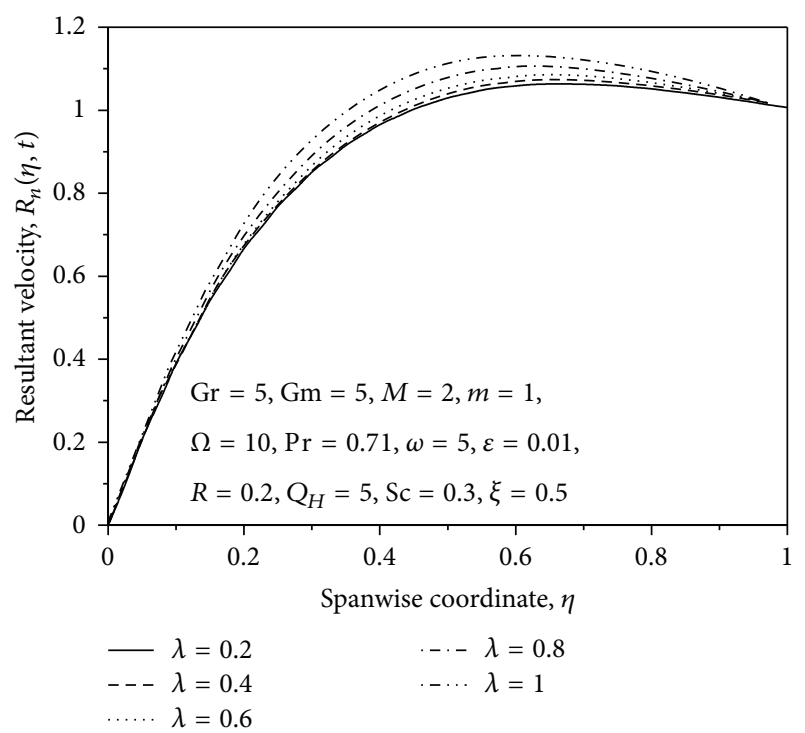

FIgURE 2: Resultant velocity $R_{n}$ due to $u$ and $v$ versus $\eta$ at $t=\pi / 4$.

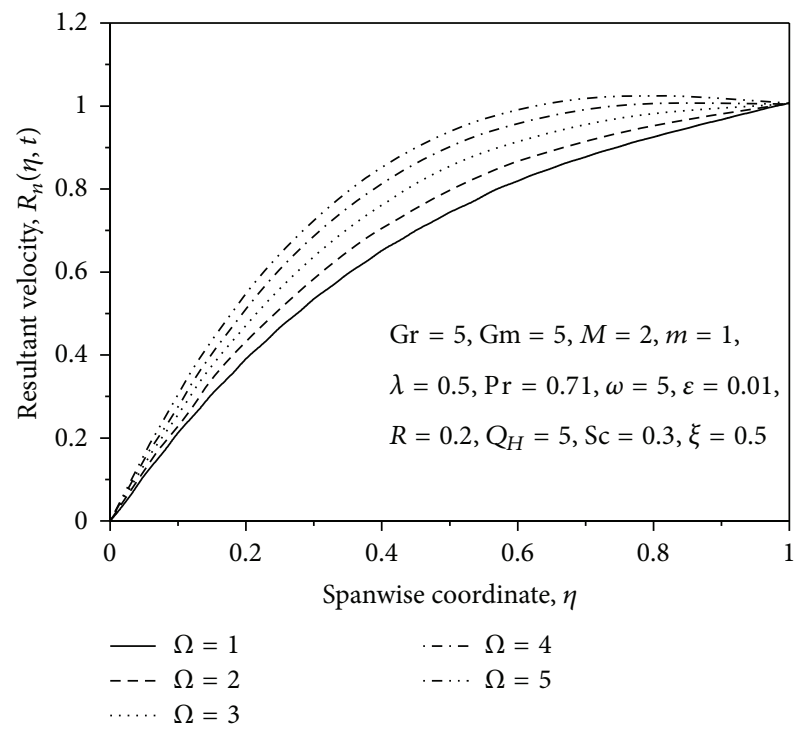

FIgURE 3: Resultant velocity $R_{n}$ due to $u$ and $v$ versus $\eta$ for small values of $\Omega$ at $t=\pi / 4$.

Figure 7 for various values of rotation parameter $\Omega$. From this figure, it is observed that the phase angle $\alpha$ decreases with an increase in rotation parameter. Figure 8 shows the variation of $\alpha$ against $\eta$ for different values of thermal Grashof number Gr, solutal Grashof number Gm, Hartmann number $M$, and Hall parameter $m$. From this figure it is found that the values of $\alpha$ decrease with an increase in the value of $\mathrm{Gr}, \mathrm{Gm}$ and $M$, whereas reverse trend is seen on the values of $\alpha$ by increasing the value of the Hall parameter $m$. The phase difference $\alpha$ for the flow is shown graphically in Figure 9 for various positive values of suction/injection parameter $\lambda$. The figure shows that the phase angle $\alpha$ decreases with the increase of suction parameter.

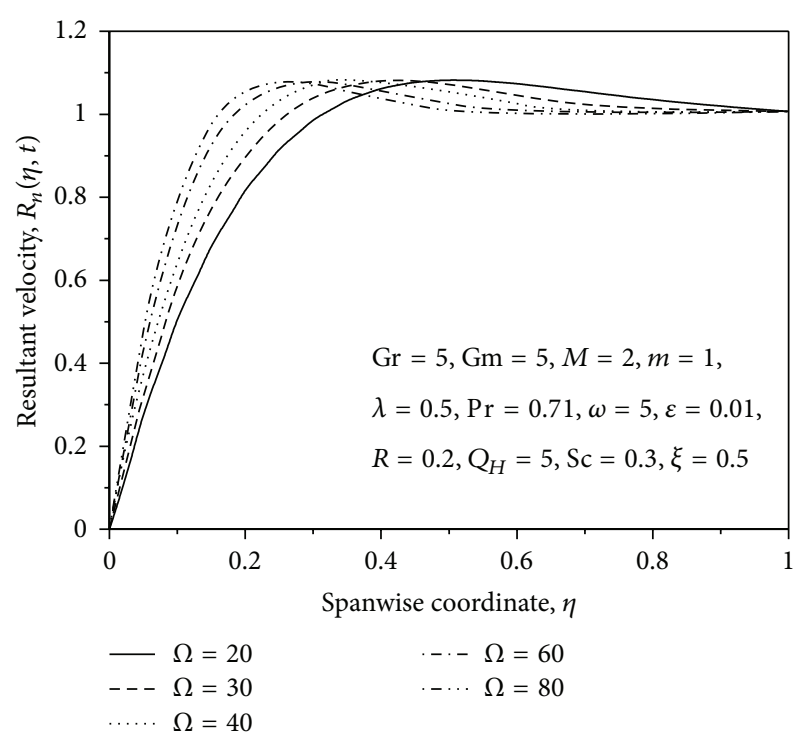

FIgURE 4: Resultant velocity $R_{n}$ due to $u$ and $v$ versus $\eta$ for large values of $\Omega$ at $t=\pi / 4$.

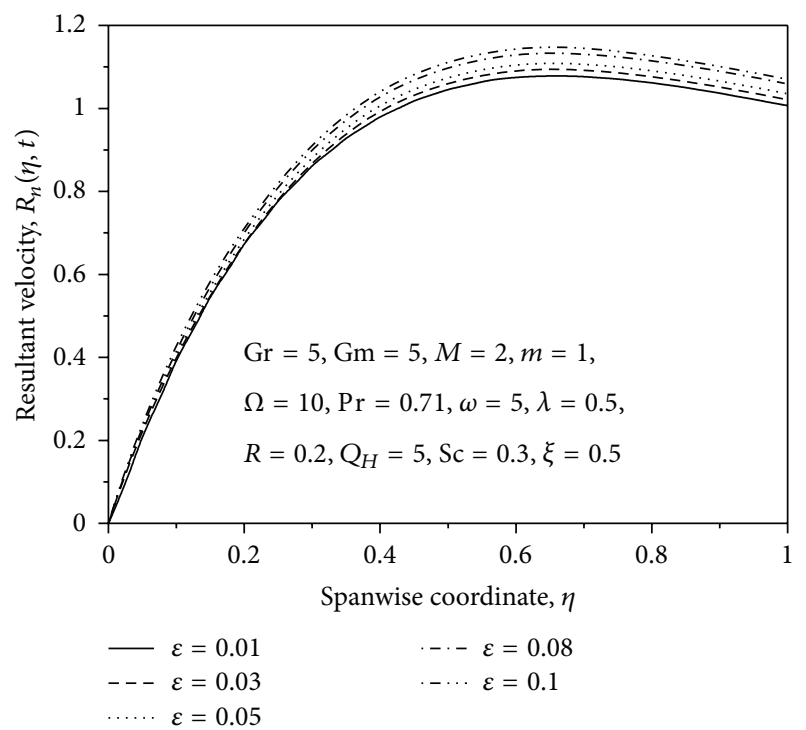

Figure 5: Resultant velocity $R_{n}$ due to $u$ and $v$ versus $\eta$ for different values of $\epsilon$ at $t=\pi / 4$.

The effect of reaction rate parameter $\xi$ on the species concentration profiles for generative chemical reaction is shown in Figure 10. It is noticed for the graph that there is a marked effect of increasing the value of the chemical reaction rate parameter $\xi$ on concentration distribution $\phi$ in the boundary layer. It is observed that increasing the value of the chemical reaction parameter $\xi$ decreases the concentration of species in the boundary layer; this is due to the fact that destructive chemical reduces the solutal boundary layer thickness and increases the mass transfer. Opposite trend is seen in the case when Schmidt number is increased as noted in Figure 11. It may also be observed from this figure that the 


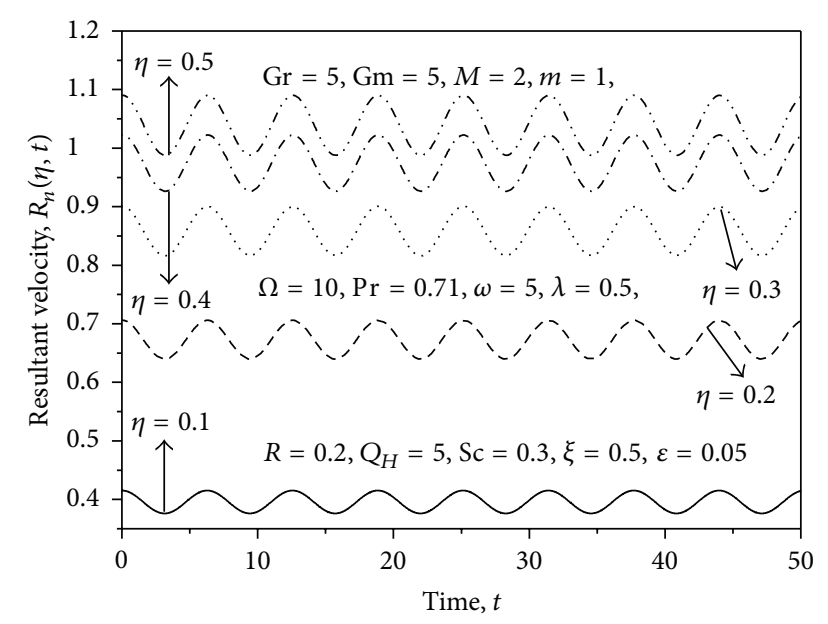

FIGURE 6: Resultant velocity $R_{n}$ due to $u$ and $v$ versus $t$ for different values of $\eta$.

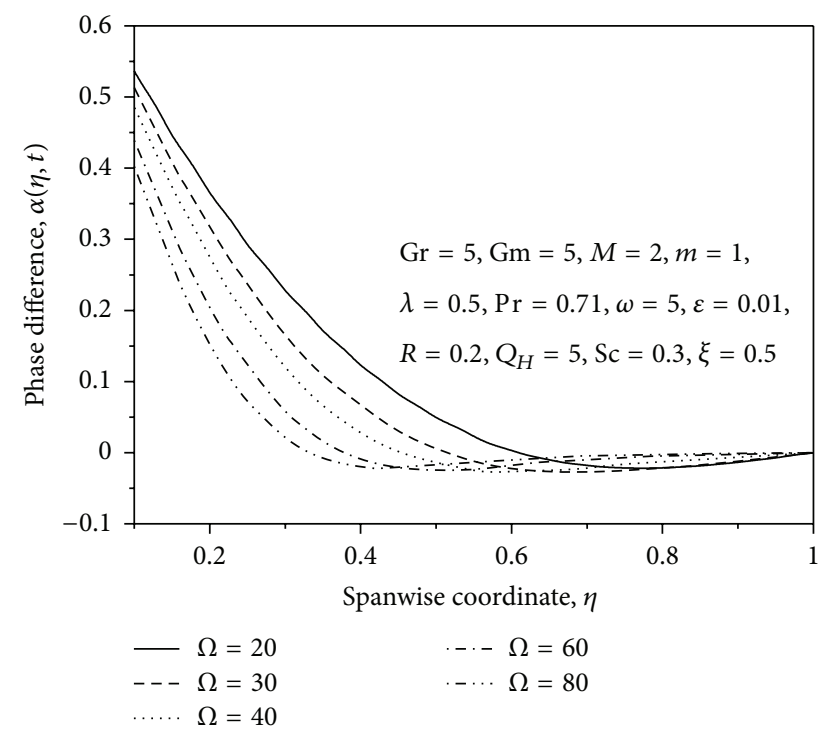

Figure 7: Phase angle $\alpha$ due to $u$ and $v$ versus $\eta$ at $t=\pi / 4$.

effect of Schmidt number Sc is to increase the concentration distribution in the solutal boundary layer.

Figure 12 has been plotted to depict the variation of temperature profiles against $\eta$ for different values of heat absorption parameter $Q_{H}$ by fixing other physical parameters. From this graph, we observe that temperature $\theta$ decreases with increase in the heat absorption parameter $Q_{H}$ because when heat is absorbed, the buoyancy force decreases the temperature profile. Figure 13 represents graph of temperature distribution with $\eta$ for different values of radiation parameter. From this figure, we note that the initial temperature $\theta=1.0$ decreases to zero satisfying the boundary condition at $\eta=1.0$. Further, it is observed from this figure that increase in the radiation parameter decreases the temperature distribution in the thermal boundary layer due to decrease in the thickness of the thermal boundary layer with thermal radiation parameter $R$. This is because large

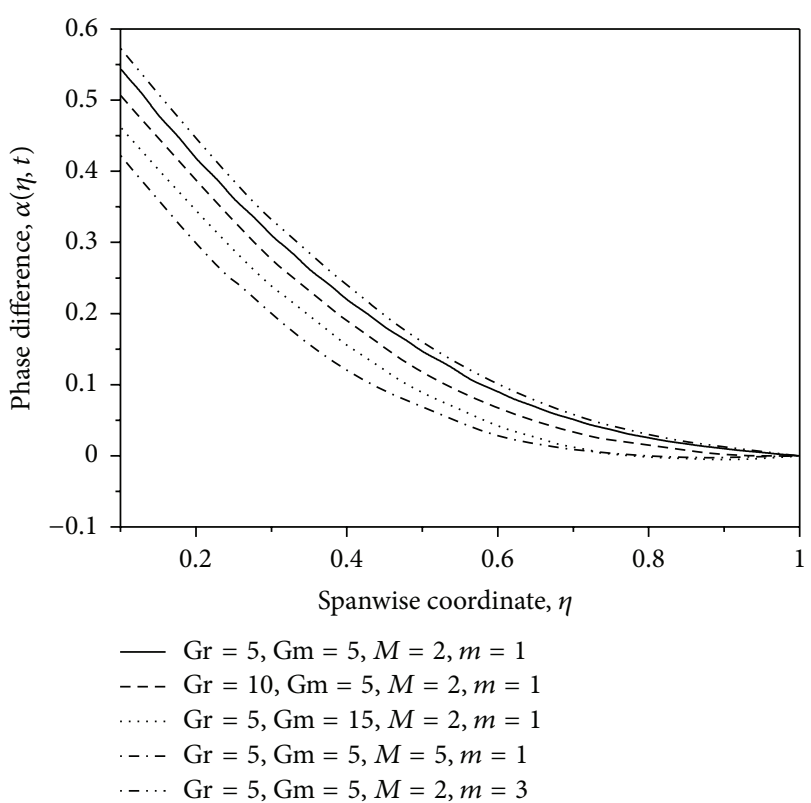

Figure 8: Phase angle $\alpha$ due to $u$ and $v$ versus $\eta$ for $\lambda=0.5, \Omega=10$, $\mathrm{Sc}=0.3, \operatorname{Pr}=0.71, R=0.2, Q_{H}=5.0, \epsilon=0.01$, and $\xi=0.5$ at $t=\pi / 4$.

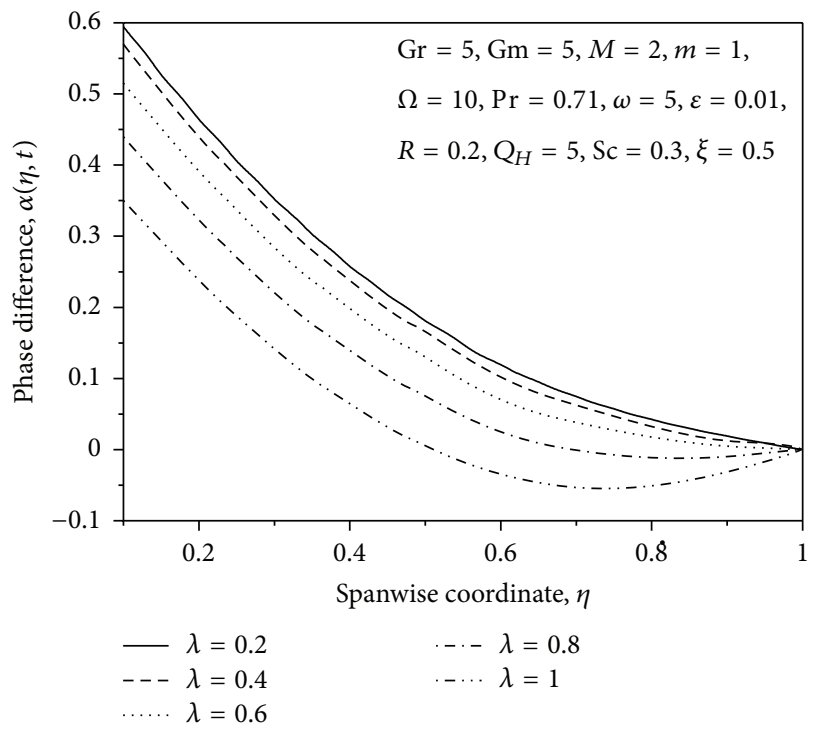

Figure 9: Phase angle $\alpha$ due to $u$ and $v$ versus $\eta$ for $\omega=5.0$ at $t=$ $\pi / 4$.

values of radiation parameter correspond to an increase in dominance of conduction over radiation, thereby decreasing the buoyancy force and the temperature in the thermal boundary layer.

Figures 14-16 show the amplitude of skin-friction, Nusselt number, and Sherwood number against frequency parameter $\omega$ for different values of $\mathrm{Gr}, Q_{H}$ and $\xi$, respectively. From Figure 14, it is observed that the skin friction increases with increasing the values of Gr. Also, the skin friction decreases slowly with increasing the value of $\omega$. The amplitude of 


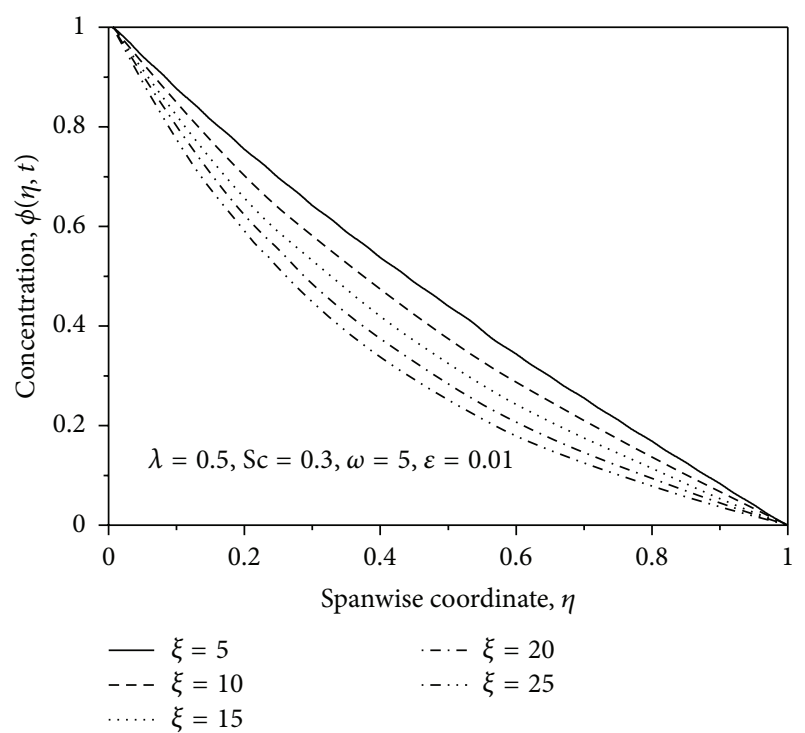

FIGURE 10: Concentration profiles against $\eta$ for different values of $\xi$ at $t=\pi / 4$.

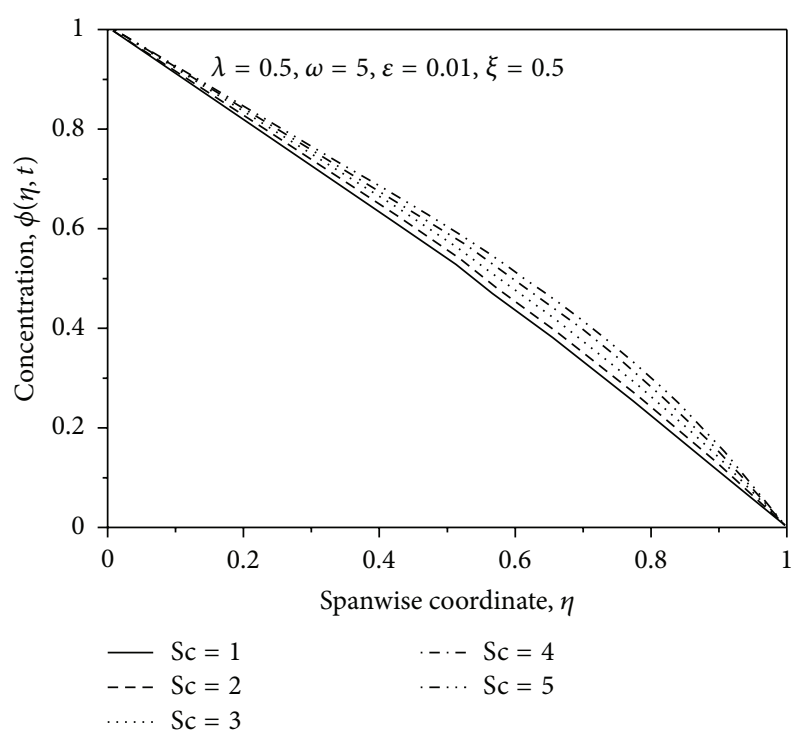

FIGURE 11: Concentration profiles against $\eta$ for different values of Sc at $t=\pi / 4$.

Nusselt number decreases with increasing the value of heat source parameter $Q_{H}$ which is shown in Figure 15 . Figure 16 shows the variation of Sherwood number with $\xi$ and $\omega$. From this figure, it is seen that the Sherwood number decreases with increasing the values of chemical reaction parameter $\xi$, and the opposite trend is seen with increasing the values of $\omega$.

\section{Conclusions}

The influence of hall current and chemical reaction on unsteady MHD heat and mass transfer of an oscillatory convective flow in a rotating vertical porous channel with

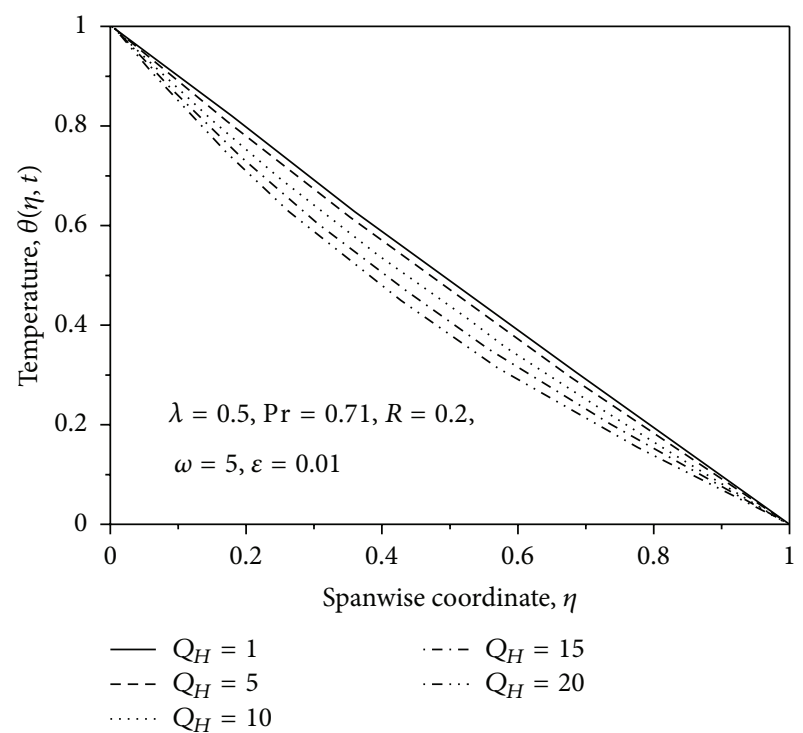

FIGURE 12: Temperature profiles against $\eta$ for different values of $Q_{H}$ at $t=\pi / 4$.

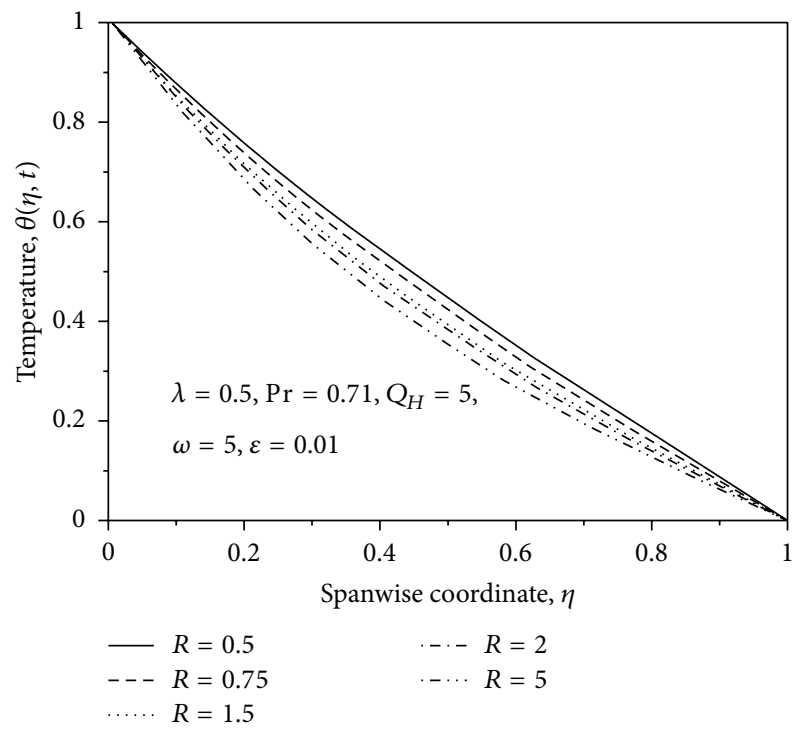

FIGURE 13: Temperature profiles against $\eta$ for different values of $R$ at $t=\pi / 4$.

thermal radiation and injection is studied analytically. Computed results are presented to exhibit their dependence on the important physical parameters. We conclude the following from the numerical results.

(i) An increase in $Q_{H}$ leads to an increase in $\tau_{0 r}, \beta_{0}, \tau_{1 r}$ and decrease in $\beta_{1}$.

(ii) An increase in radiation parameter $R$ and chemical reaction parameter $\xi$ leads to increase in $\tau_{0 r}, \beta_{0}$, and $\beta_{1}$ but decrease in $\tau_{1 r}$.

(iii) An increase in Gr, Gm, $M, \Omega$, and $\lambda$ leads to decrease in $\alpha_{0}$ and $\alpha_{1}$, whereas reverse effect is seen by increasing Hall parameter $m$. 


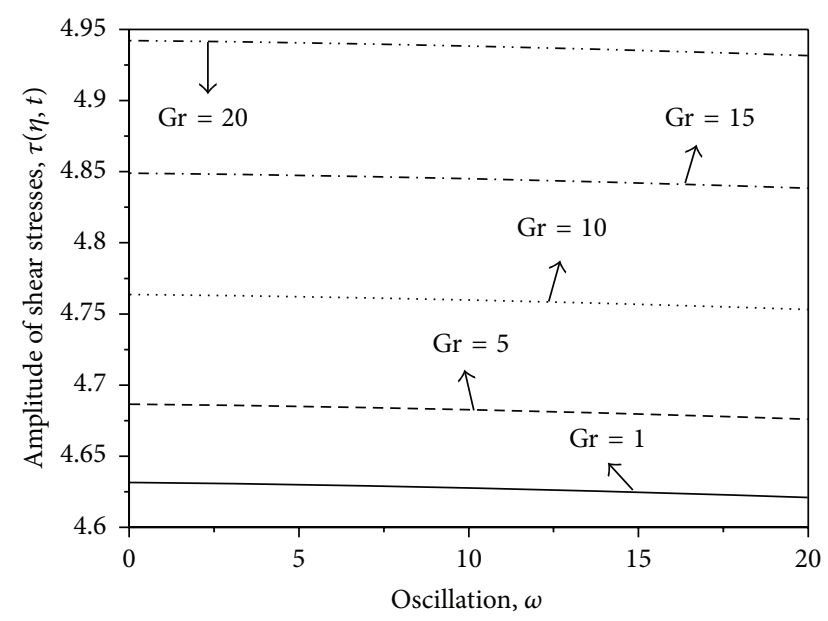

FIGURE 14: Skin friction coefficient against $\omega$ for different values of Gr with $\mathrm{Gm}=5.0, M=2.0, m=1.0, \Omega=10.0, \operatorname{Pr}=0.71, R=0.2$, $Q_{H}=5.0, \mathrm{Sc}=0.3, \xi=0.5, \epsilon=0.01$ at $t=\pi / 4$.

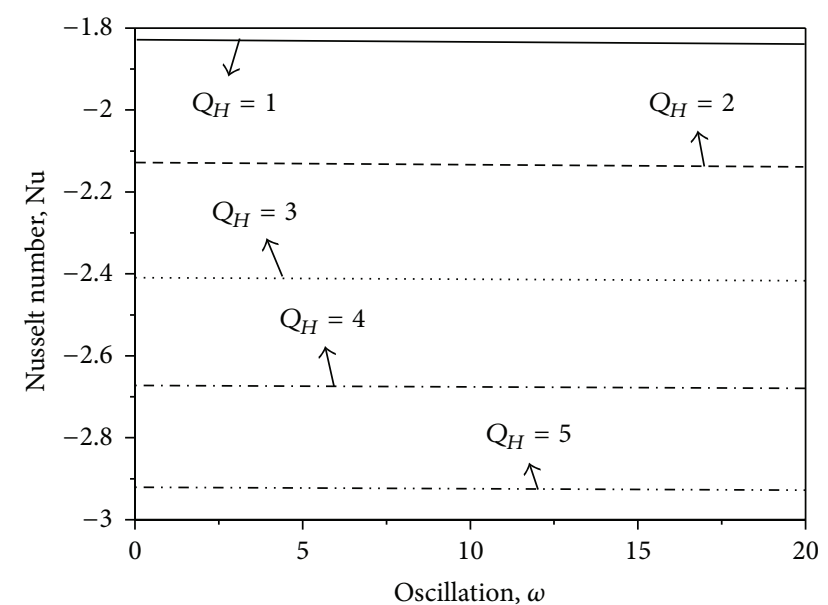

FIGURE 15: Nusselt number against $\omega$ for different values of $Q_{H}$ with $\lambda=0.5, \operatorname{Pr}=0.71, R=2.0, \epsilon=0.01$ at $t=\pi / 4$.

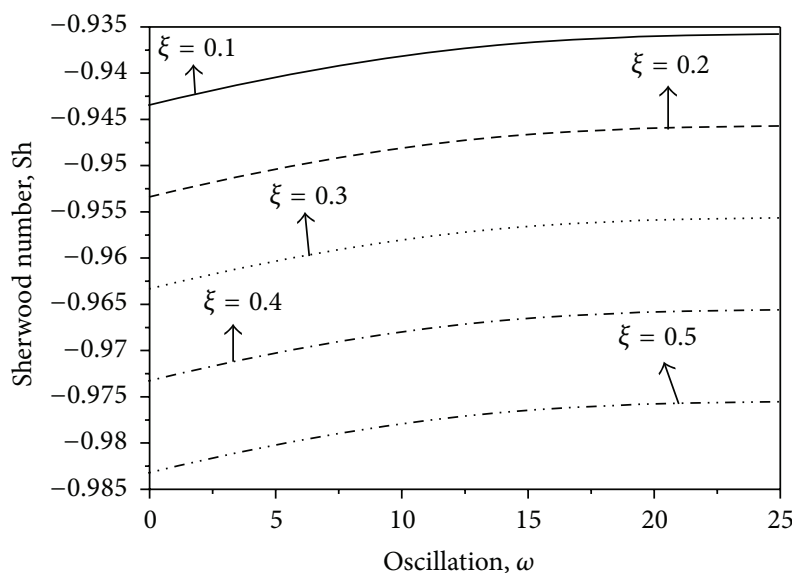

FIGURE 16: Sherwood number against $\omega$ for different values of $\xi$ with $\lambda=0.5, \epsilon=0.01, \mathrm{Sc}=0.3$ at $t=\pi / 4$. (iv) The amplitude $R_{n}$ increases with the increase of $\lambda$ and $\Omega$.

(v) The value of $R_{n}$ decreases with the increase in chemical reaction parameter $\xi$ and oscillation parameter $\omega$.

(vi) The skin friction increases with increase in thermal Grashof number Gr.

\section{Appendix}

Consider the following:

$$
\begin{aligned}
& R_{1}=\frac{3 R}{3 R+4}, \quad R_{2}=R_{1} \operatorname{Pr} \\
& h_{1}=\frac{\operatorname{Sc} \lambda+\sqrt{(\operatorname{Sc} \lambda)^{2}+4 \xi \operatorname{Sc}}}{2} \text {, } \\
& h_{2}=\frac{\operatorname{Sc} \lambda-\sqrt{(\mathrm{Sc} \lambda)^{2}+4 \xi \mathrm{Sc}}}{2} \text {, } \\
& h_{3}=\frac{\operatorname{Sc} \lambda+\sqrt{(\operatorname{Sc} \lambda)^{2}+4(i \omega+\xi) S c}}{2} \text {, } \\
& h_{4}=\frac{\operatorname{Sc} \lambda-\sqrt{(\operatorname{Sc} \lambda)^{2}+4(i \omega+\xi) \mathrm{Sc}}}{2} \text {, } \\
& h_{5}=\frac{\operatorname{Sc} \lambda+\sqrt{(\operatorname{Sc} \lambda)^{2}-4(i \omega-\xi) S c}}{2} \text {, } \\
& h_{6}=\frac{\operatorname{Sc} \lambda-\sqrt{(\operatorname{Sc} \lambda)^{2}-4(i \omega-\xi) S c}}{2} \text {, } \\
& h_{7}=\frac{R_{2} \lambda+\sqrt{\left(R_{2} \lambda\right)^{2}+4 R_{1} Q_{H}}}{2}, \\
& h_{8}=\frac{R_{2} \lambda-\sqrt{\left(R_{2} \lambda\right)^{2}+4 R_{1} Q_{H}}}{2} \text {, } \\
& h_{9}=\frac{R_{2} \lambda+\sqrt{\left(R_{2} \lambda\right)^{2}+4 R_{1}\left(i \omega \operatorname{Pr}+Q_{H}\right)}}{2} \text {, } \\
& h_{10}=\frac{R_{2} \lambda-\sqrt{\left(R_{2} \lambda\right)^{2}+4 R_{1}\left(i \omega \operatorname{Pr}+Q_{H}\right)}}{2} \text {, } \\
& h_{11}=\frac{R_{2} \lambda+\sqrt{\left(R_{2} \lambda\right)^{2}-4 R_{1}\left(i \omega \operatorname{Pr}-Q_{H}\right)}}{2} \text {, } \\
& h_{12}=\frac{R_{2} \lambda-\sqrt{\left(R_{2} \lambda\right)^{2}-4 R_{1}\left(i w \operatorname{Pr}+Q_{H}\right)}}{2} \text {, } \\
& h_{13}=\frac{\lambda+\sqrt{\lambda^{2}+4 S}}{2}, \quad h_{14}=\frac{\lambda-\sqrt{\lambda^{2}+4 S}}{2} \text {, }
\end{aligned}
$$




$$
\begin{aligned}
& h_{15}=\frac{\lambda+\sqrt{\lambda^{2}+4(S+i \omega)}}{2}, \\
& h_{16}=\frac{\lambda-\sqrt{\lambda^{2}+4(S+i \omega)}}{2}, \\
& h_{17}=\frac{\lambda+\sqrt{\lambda^{2}+4(S-i \omega)}}{2}, \\
& h_{18}=\frac{\lambda-\sqrt{\lambda^{2}+4(S-i \omega)}}{2}, \\
& A_{1}=\frac{\lambda^{2} \mathrm{Gr} e^{h_{7}}}{\left(e^{h_{7}}-e^{h_{8}}\right)\left[h_{8}^{2}-\lambda h_{8}-S\right]}, \\
& A_{2}=\frac{\lambda^{2} \mathrm{Gr} e^{h_{8}}}{\left(e^{h_{7}}-e^{h_{8}}\right)\left[h_{7}^{2}-\lambda h_{7}-S\right]}, \\
& A_{3}=\frac{\lambda^{2} \mathrm{Gr} e^{h_{1}}}{\left(e^{h_{1}}-e^{h_{2}}\right)\left[h_{2}^{2}-\lambda h_{2}-S\right]}, \\
& A_{4}=\frac{\lambda^{2} \mathrm{Gr} e^{h_{2}}}{\left(e^{h_{1}}-e^{h_{2}}\right)\left[h_{1}^{2}-\lambda h_{1}-S\right]}, \\
& A_{5}=\frac{\lambda^{2} \mathrm{Gr} e^{h_{9}}}{\left(e^{h_{9}}-e^{h_{10}}\right)\left[h_{10}^{2}-\lambda h_{10}-(S+i \omega)\right]}, \\
& A_{6}=\frac{\lambda^{2} \mathrm{Gr} e^{h_{10}}}{\left(e^{h_{9}}-e^{h_{10}}\right)\left[h_{9}^{2}-\lambda h_{9}-(S+i \omega)\right]}, \\
& A_{7}=\frac{\lambda^{2} \mathrm{Gm} e^{h_{3}}}{\left(e^{h_{3}}-e^{h_{4}}\right)\left[h_{4}^{2}-\lambda h_{4}-(S+i \omega)\right]}, \\
& A_{8}=\frac{\lambda^{2} G m e^{h_{4}}}{\left(e^{h_{3}}-e^{h_{4}}\right)\left[h_{3}^{2}-\lambda h_{3}-(S+i \omega)\right]}, \\
& A_{9}=\frac{\lambda^{2} \mathrm{Gr} e^{h_{11}}}{\left(e^{h_{11}}-e^{h_{12}}\right)\left[h_{12}^{2}-\lambda h_{12}-(S-i \omega)\right]}, \\
& A_{10}=\frac{\lambda^{2} \mathrm{Gr} e^{h_{12}}}{\left(e^{h_{11}}-e^{h_{12}}\right)\left[h_{11}^{2}-\lambda h_{11}-(S-i \omega)\right]}, \\
& A_{11}=\frac{\lambda^{2} \mathrm{Gm} e^{h_{5}}}{\left(e^{h_{5}}-e^{h_{6}}\right)\left[h_{6}^{2}-\lambda h_{6}-(S-i \omega)\right]}, \\
& A_{12}=\frac{\lambda^{2} \mathrm{Gm} e^{h_{6}}}{\left(e^{h_{5}}-e^{h_{6}}\right)\left[h_{5}^{2}-\lambda h_{5}-(S-i \omega)\right]} .
\end{aligned}
$$

\section{Nomenclature}

$C^{*}$ : Dimensional concentration

$C_{0}$ : Concentration at the left plate

$C_{d}$ : Concentration at the right plate

$c_{p}$ : Specific heat at constant pressure

$d$ : Distance of the plates

$D_{m}$ : Chemical molecular diffusivity

$e$ : Electric charge $g_{0}:$

Modified Grashof number for mass transfer

Gr: $\quad$ Modified Grashof number for heat transfer

$\vec{H}: \quad$ Magnetic field

$H_{0}$ : $\quad$ Magnetic field of uniform strength

$H_{x}: \quad x$-component of magnetic field

$\vec{J}: \quad$ Current density

$J_{x}: \quad x$-component of current density

$k^{*}: \quad$ Mean absorption coefficient

$k_{1}$ : $\quad$ Chemical reaction rate constant

$m: \quad$ Hall parameter

$M: \quad$ Hartmann number

Nu: $\quad$ Nusselt number

$n_{e}: \quad \quad$ Number density of the electron

$P^{*}: \quad$ Dimensional pressure

$p_{e}: \quad$ Electron pressure

Pr: $\quad$ Prandtl number

$q_{r}^{*}: \quad$ Radiative heat flux

$Q_{0}: \quad$ Dimensional heat source

$Q_{H}: \quad$ Heat source parameter

$R: \quad$ Radiation parameter

$R_{0}: \quad$ Amplitude for steady flow

$R_{n}: \quad$ Resultant velocity

$R_{v}: \quad$ Amplitude for unsteady flow

Sc: $\quad$ Schmidt number

Sh: $\quad$ Sherwood number

$t^{*}$ : $\quad$ Dimensional time

$T^{*}: \quad$ Dimensional temperature

$T_{0}$ : $\quad$ Temperature at the left wall

$T_{d}: \quad$ Temperature at the right wall

$U_{0}$ : $\quad$ Nonzero constant mean velocity

$u_{0}$ : Primary velocity component for steady flow

$u_{1}$ : $\quad$ Primary velocity component for unsteady

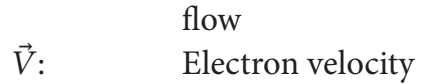

$v_{0}$ : Secondary velocity component for steady

$v_{1}$ : $\quad$ Secondary velocity component for unsteady

$v_{1}: \quad$ flow

$u^{*}, v^{*}, w^{*}$ : Velocity components are in the $x^{*}-, y^{*}$, $z^{*}$-directions, respectively

$w_{0}$ : Dimensional injection/suction velocity.

Greek Symbols

$\alpha_{0}$ : Phase difference for steady flow

$\alpha_{1}$ : Phase difference for unsteady flow

$\alpha$ : Phase difference of the flow

$\beta$ : Coefficient of thermal expansion

$\beta^{*}$ : Coefficient of solutal expansion

$\beta_{0}$ : Phase difference of shear stresses for the steady flow

$\beta_{1}$ : Phase difference of shear stresses for the unsteady flow

$\beta_{2}$ : Phase difference of shear stresses for the flow

$\delta$ : $\quad$ Phase difference of mass flux

$\epsilon$ : Small positive constant

$\eta$ : Dimensionless distance

$\gamma$ : Phase difference of heat flux 
$\kappa: \quad$ Fluid thermal conductivity

$\lambda$ : Injection/suction parameter

$\mu$ : Dynamic viscosity

$\mu_{e}:$ Magnetic permeability

$\nu$ : Kinematic viscosity

$\omega:$ Oscillation parameter

$\Omega^{*}$ : Dimensional angular velocity

$\Omega$ : Angular velocity

$\omega_{e}$ : Cyclotron frequency

$\Phi:$ Amplitude of mass flux

$\phi$ : Nondimensional concentration

$\rho:$ Density

$\sigma: \quad$ Electric conductivity

$\sigma^{*}$ : Stefan-Boltzmann constant

$\tau$ : Amplitude of shear stresses for the flow

$\tau_{0 r}$ : Amplitude of shear stresses for the steady flow

$\tau_{1 r}:$ Amplitude of shear stresses for the unsteady flow

$\tau_{e}:$ Electron collision time

$\theta: \quad$ Non-dimensional temperature

$\Theta$ : Amplitude of heat flux.

\section{Acknowledgment}

One of the authors (Dulal Pal) is grateful to the University Grants Commission (UGC), New Delhi, for providing financial support under SAP-DRS (Phase-II) Grant.

\section{References}

[1] M. A. El-Hakiem, "MHD oscillatory flow on free convectionradiation through a porous medium with constant suction velocity," Journal of Magnetism and Magnetic Materials, vol. 220, no. 2-3, pp. 271-276, 2000.

[2] B. S. Jaiswal and V. M. Soundalgekar, "Oscillating plate temperature effects on a flow past an infinite vertical porous plate with constant suction and embedded in a porous medium," Heat and Mass Transfer, vol. 37, no. 2-3, pp. 125-131, 2001.

[3] K. D. Singh, M. G. Gorla, and H. Raj, "A periodic solution of oscillatory couette flow through porous medium in rotating system," Indian Journal of Pure and Applied Mathematics, vol. 36, no. 3, pp. 151-159, 2005.

[4] D. Pal and I. S. Shivakumara, "Mixed convection heat transfer from a vertical heated plate embedded in a sparsely packed porous medium," International Journal of Applied Mechanics and Engineering, vol. 11, no. 4, pp. 929-939, 2006.

[5] T. G. Cowling, Magnetohydrodynamics, Interscience Publishers, New York, NY, USA, 1957.

[6] A. S. Gupta, "Hydromagnetic flow past a porous flat plate with hall effects," Acta Mechanica, vol. 22, no. 3-4, pp. 281-287, 1975.

[7] R. N. Jana, A. S. Gupta, and N. Datta, "Hall effects on the hydro magnetic flow past an infinite porous flat plate," Journal of the Physical Society of Japan, vol. 43, no. 5, pp. 1767-1772, 1977.

[8] O. D. Makinde and P. Y. Mhone, "Heat transfer to MHD oscillatory flow in a channel filled with porous medium," Romanian Journal of Physics, vol. 50, pp. 931-938, 2005.

[9] Z. Zhang and J. Wang, "On the similarity solutions of magnetohydrodynamic flows of power-law fluids over a stretching sheet," Journal of Mathematical Analysis and Applications, vol. 330, no. 1, pp. 207-220, 2007.

[10] M. Hameed and S. Nadeem, "Unsteady MHD flow of a nonNewtonian fluid on a porous plate," Journal of Mathematical Analysis and Applications, vol. 325, no. 1, pp. 724-733, 2007.

[11] O. D. Makinde, O. A. Beg, and H. S. Takhar, "Magnetohydrodynamic viscous flow in a rotating porous medium cylindrical annalus with on applied radial magnetic field," International Journal of Applied Mathematics and Mechanics, vol. 5, pp. 6881, 2009.

[12] P. Sibanda and O. D. Makinde, "On steady MHD flow and heat transfer past a rotating disk in a porous medium with ohmic heating and viscous dissipation," International Journal of Numerical Methods for Heat and Fluid Flow, vol. 20, no. 3, pp. 269-285, 2010.

[13] I. Pop and T. Watanabe, "Hall effects on magnetohydrodynamic free convection about a semi-infinite vertical flat plate," International Journal of Engineering Science, vol. 32, no. 12, pp. 19031911, 1994.

[14] L. K. Saha, S. Siddiqa, and M. A. Hossain, "Effect of Hall current on MHD natural convection flow from vertical permeable flat plate with uniform surface heat flux," Applied Mathematics and Mechanics (English Edition), vol. 32, no. 9, pp. 1127-1146, 2011.

[15] D. Pal, B. Talukdar, I. S. Shivakumara, and K. Vajravelu, "Effects of Hall current and chemical reaction on oscillatory mixed convection-radiation of a micropolar fluid in a rotating system," Chemical Engineering Communications, vol. 199, pp. 943-965, 2012.

[16] A. C. Cogley, W. C. Vincenti, and S. E. Gilles, "Differential approximation for radiation transfer in a nongray gas near equilibrium," American Institute of Aeronautics and Astronautics Journal, vol. 6, pp. 551-555, 1968.

[17] M. A. Mansour, "Radiative and free-convection effects on the oscillatory flow past a vertical plate," Astrophysics and Space Science, vol. 166, no. 2, pp. 269-275, 1990.

[18] M. A. Hossain and H. S. Takhar, "Radiation effect on mixed convection along a vertical plate with uniform surface temperature," Heat and Mass Transfer, vol. 31, no. 4, pp. 243-248, 1996.

[19] M. A. Hossain, M. A. Alim, and D. A. S. Rees, "The effect of radiation on free convection from a porous vertical plate," International Journal of Heat and Mass Transfer, vol. 42, no. 1, pp. 181-191, 1999.

[20] M. A. Seddeek, "Effects of radiation and variable viscosity on a MHD free convection flow past a semi-infinite flat plate with an aligned magnetic field in the case of unsteady flow," International Journal of Heat and Mass Transfer, vol. 45, no. 4, pp. 931-935, 2002.

[21] R. Muthucumaraswamy and G. K. Senthil, "Studied the effect of heat and mass transfer on moving vertical plate in the presence of thermal radiation," Journal of Theoretical And Applied Mechanics, vol. 31, no. 1, pp. 35-46, 2004.

[22] D. Pal, "Heat and mass transfer in stagnation-point flow towards a stretching surface in the presence of buoyancy force and thermal radiation," Meccanica, vol. 44, no. 2, pp. 145-158, 2009.

[23] O. Aydin and A. Kaya, "Radiation effect on MHD mixed convection flow about a permeable vertical plate," Heat and Mass Transfer, vol. 45, no. 2, pp. 239-246, 2008.

[24] R. A. Mohamed, "Double-diffusive convection-radiation interaction on unsteady MHD flow over a vertical moving porous plate with heat generation and Soret effects," Applied Mathematical Sciences, vol. 3, no. 13-16, pp. 629-651, 2009. 
[25] D. S. Chauhan and P. Rastogi, "Radiation effects on natural convection MHD flow in a rotating vertical porous channel partially filled with a porous medium," Applied Mathematical Sciences, vol. 4, no. 13-16, pp. 643-655, 2010.

[26] S. Y. Ibrahim and O. D. Makinde, "Radiation effect on chemically reacting magnetohydrodynamics (MHD) boundary layer flow of heat and mass transfer through a porous vertical flat plate," International Journal of Physical Sciences, vol. 6, no. 6, pp. 1508-1516, 2011.

[27] D. Pal and H. Mondal, "The influence of thermal radiation on hydromagnetic darcy-forchheimer mixed convection flow past a stretching sheet embedded in a porous medium," Meccanica, vol. 46, no. 4, pp. 739-753, 2011.

[28] G. Palani and K. Y. Kim, "Influence of magnetic field and thermal radiation by natural convection past vertical cone subjected to variable surface heat flux," Applied Mathematics and Mechanics (English Edition), vol. 33, pp. 605-620, 2012.

[29] M. A. A. Mahmoud and S. E. Waheed, "Variable fluid properties and ther-28 mal radiation effects on flow and heat transfer in micropolar fluid film past moving permeable infinite flat plate with slip velocity," Applied Mathematics and Mechanics (English Edition), vol. 33, pp. 663-678, 2012.

[30] E. M. Aboeldahab and E. M. E. Elbarbary, "Hall current effect on magnetohydrodynamic free-convection flow past a semiinfinite vertical plate with mass transfer," International Journal of Engineering Science, vol. 39, no. 14, pp. 1641-1652, 2001.

[31] E. M. Abo-Eldahab and M. A. El Aziz, "Viscous dissipation and Joule heating effects on MHD-free convection from a vertical plate with power-law variation in surface temperature in the presence of Hall and ion-slip currents," Applied Mathematical Modelling, vol. 29, no. 6, pp. 579-595, 2005.

[32] R. Kandasamy, K. Periasamy, and K. K. Sivagnana Prabhu, "Chemical reaction, heat and mass transfer on MHD flow over a vertical stretching surface with heat source and thermal stratification effects," International Journal of Heat and Mass Transfer, vol. 48, no. 21-22, pp. 4557-4561, 2005.

[33] R. Muthucumaraswamy and B. Janakiraman, "Mass transfer effects on isothermal vertical oscillating plate in the presence of chemical reaction," International Journal of Applied Mathematics and Mechanics, vol. 4, no. 1, pp. 66-74, 2008.

[34] P. R. Sharma and K. D. Singh, "Unsteady MHD free convective flow and heat transfer along a vertical porous plate with variable suction and internal heat generation," International Journal of Applied Mathematics and Mechanics, vol. 4, no. 5, pp. 1-8, 2009.

[35] M. Sudheer Babu and P. V. Satya Narayan, "Effects of the chemical reaction and radiation absorption on free convection flow through porous medium with variable suction in the presence of uniform magnetic field," Journal of Heat and Mass Transfer, vol. 3, pp. 219-234, 2009.

[36] O. D. Makinde and T. Chinyoka, "Numerical study of unsteady hydromagnetic Generalized Couette flow of a reactive thirdgrade fluid with asymmetric convective cooling," Computers and Mathematics with Applications, vol. 61, no. 4, pp. 1167-1179, 2011.

[37] D. Pal and B. Talukdar, "Combined effects of Joule heating and chemical reaction on unsteady magnetohydrodynamic mixed convection of a viscous dissipating fluid over a vertical plate in porous media with thermal radiation," Mathematical and Computer Modelling, vol. 54, no. 11-12, pp. 3016-3036, 2011.

[38] K. D. Singh and R. Kumar, "Combined effects of hall current and rotation on free convection MHD flow in a porous channel,"
Indian Journal of Pure and Applied Physics, vol. 47, no. 9, pp. 617623, 2009.

[39] R. C. Meyer, "On reducing aerodynamic heat transfer rates by magnetohydrodynamic techniques," Journal of the Aerospace Sciences, vol. 25, p. 561, 1958. 


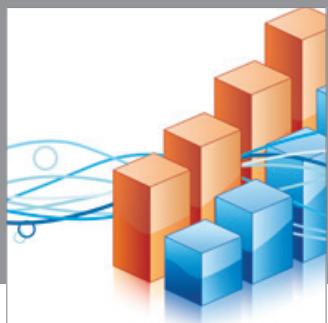

Advances in

Operations Research

mansans

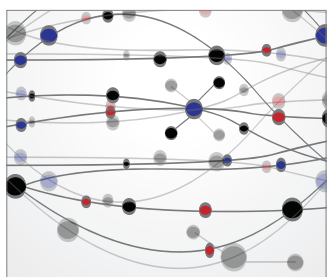

The Scientific World Journal
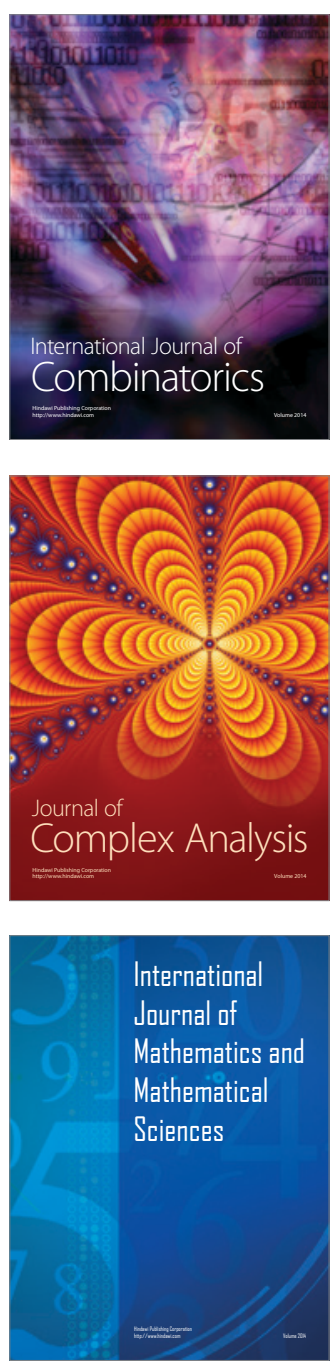
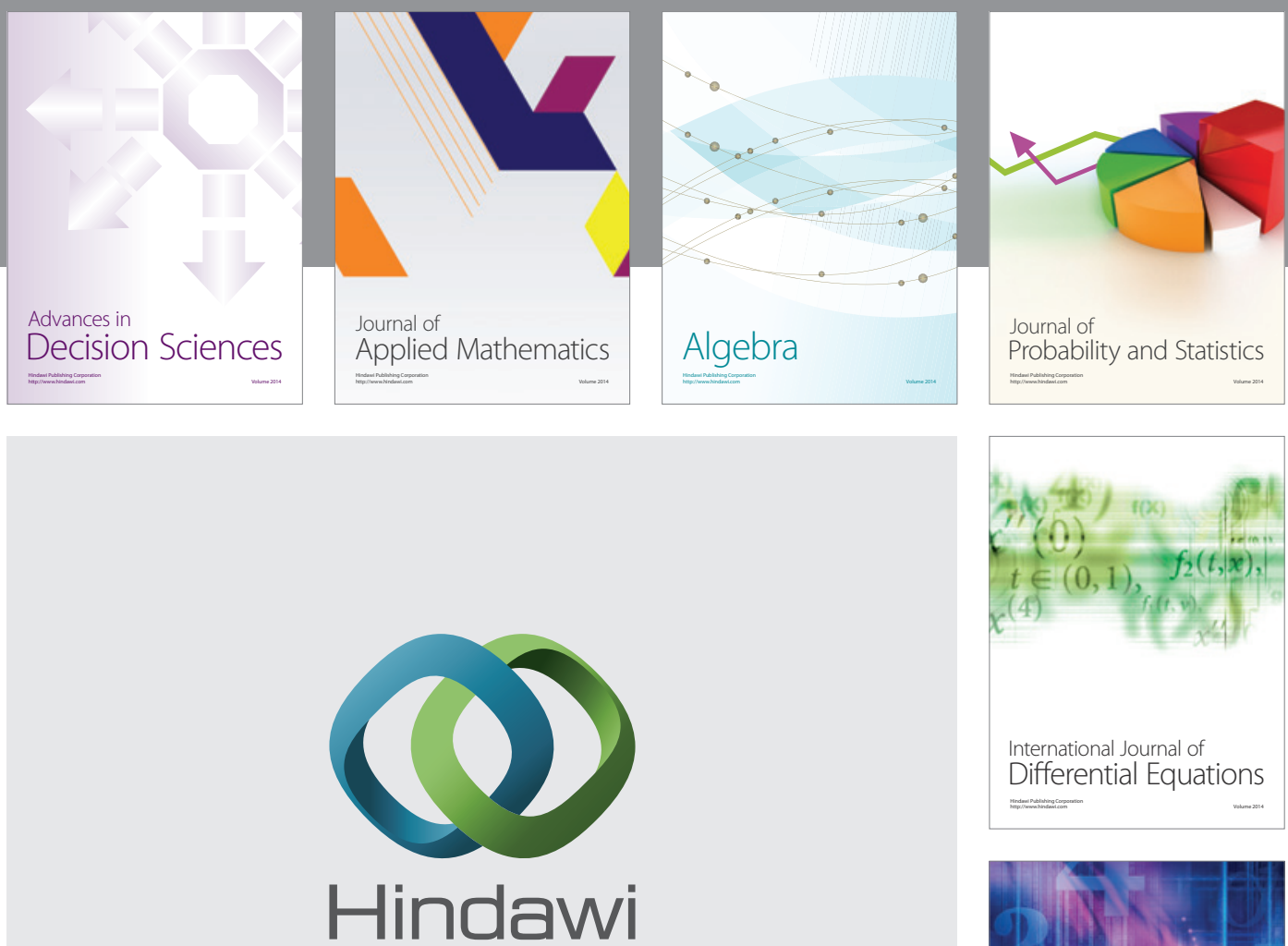

Submit your manuscripts at http://www.hindawi.com
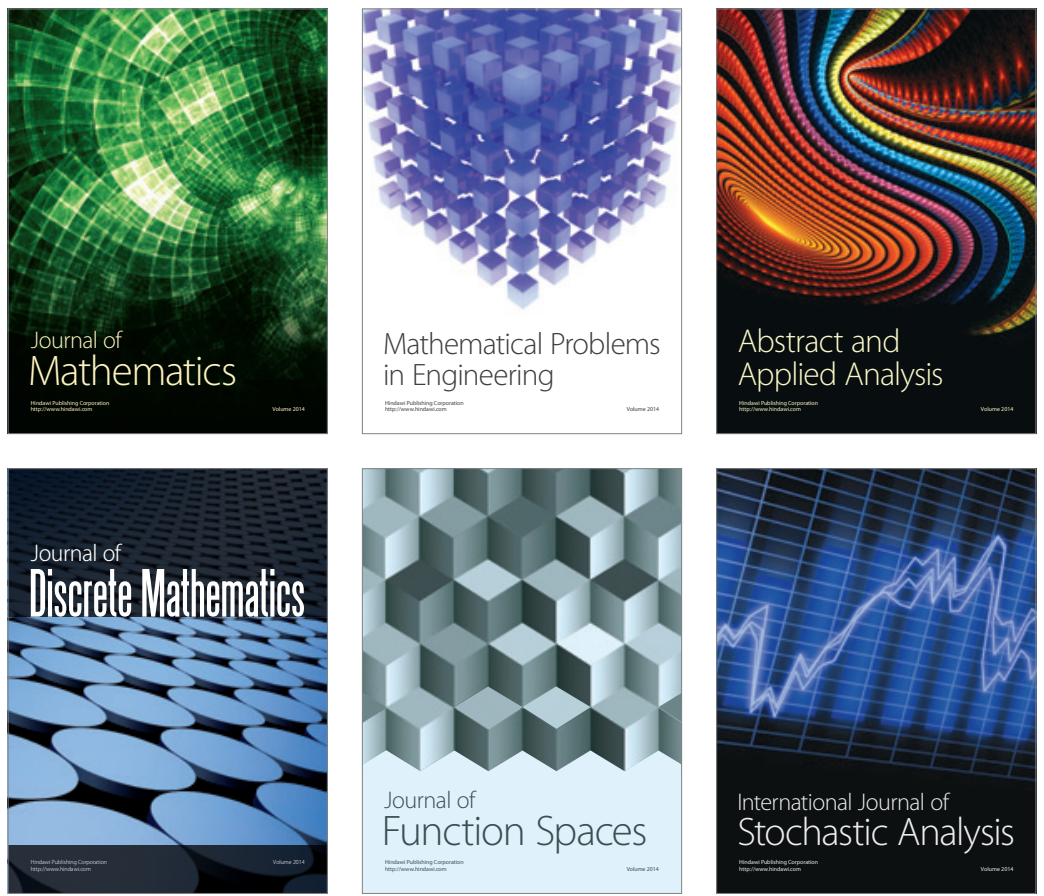

Journal of

Function Spaces

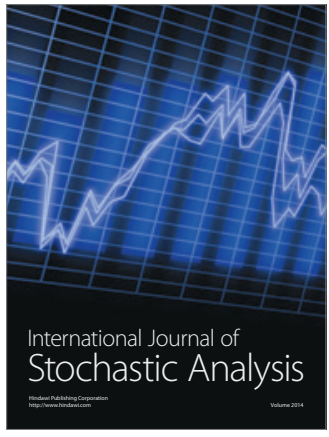

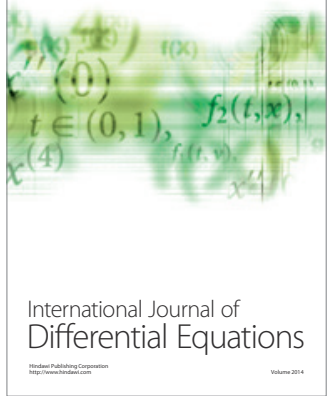
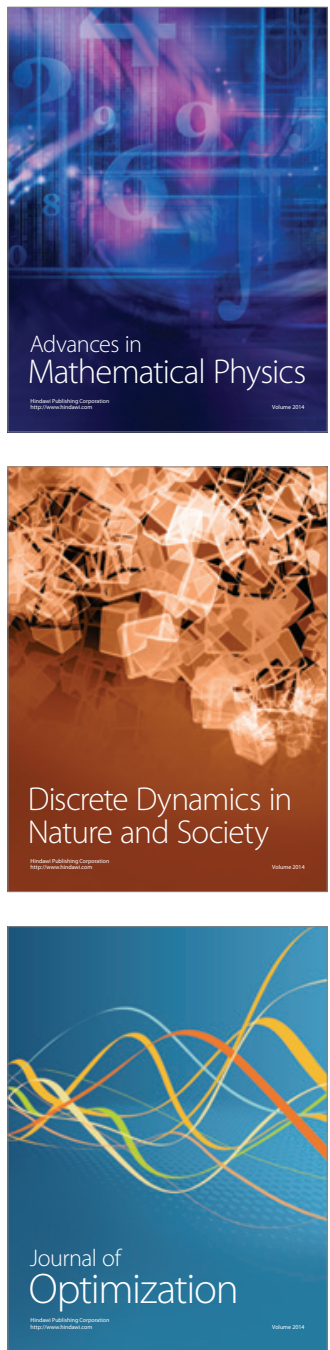